Article

\title{
Velocity Estimation of Ocean Surface Currents in along-Track InSAR System Based on Conditional Generative Adversarial Networks
}

\author{
He Yan*, Qianru Hou, Guodong Jin, Xing Xu, Gong Zhang and Daiyin Zhu \\ College of Electronic and Information Engineering, Nanjing University of Aeronautics and Astronautics, \\ Nanjing 210016, China; houqianru@nuaa.edu.cn (Q.H.); jinguodong@nuaa.edu.cn (G.J.); \\ xuxing@nuaa.edu.cn (X.X.); gzhang@nuaa.edu.cn (G.Z.); zhudy@nuaa.edu.cn (D.Z.) \\ * Correspondence: yanhe@nuaa.edu.cn
}

Citation: Yan, H.; Hou, Q.; Jin, G.; Xu, X.; Zhang, G.; Zhu, D. Velocity Estimation of Ocean Surface Currents in along-Track InSAR System Based on Conditional Generative Adversarial Networks. Remote Sens. 2021, 13, 4088. https://doi.org/ $10.3390 /$ rs13204088

Academic Editor: Weimin Huang

Received: 5 September 2021

Accepted: 11 October 2021

Published: 13 October 202

Publisher's Note: MDPI stays neutral with regard to jurisdictional claims in published maps and institutional affiliations.

Copyright: (c) 2021 by the authors. Licensee MDPI, Basel, Switzerland This article is an open access article distributed under the terms and conditions of the Creative Commons Attribution (CC BY) license (https:// creativecommons.org/licenses/by/ $4.0 /)$.

\begin{abstract}
Velocity estimation of ocean surface currents is of great significance in the fields of the fishery, shipping, sewage discharge, and military affairs. Over the last decade, along-track interferometric synthetic aperture radar (along-track InSAR) has been demonstrated to be one of the important instruments for large-area and high-resolution ocean surface current velocity estimation. The calculation method of the traditional ocean surface current velocity, as influenced by the large-scale wave orbital velocity and the Bragg wave phase velocity, cannot easily separate the current velocity, characterized by large error and low efficiency. In this paper, a novel velocity estimation method of ocean surface currents is proposed based on Conditional Generative Adversarial Networks (CGANs). The main processing steps are as follows: firstly, the known ocean surface current field diagrams and their corresponding interferometric phase diagrams are constructed as the training dataset; secondly, the estimation model of the ocean surface current field is constructed based on the pix2pix algorithm and trained by the training dataset; finally, the interferometric phase diagrams in the test dataset are input into the trained model. In the simulation experiment, processing results of the proposed method are compared with those of traditional ocean surface current velocity estimation methods, which demonstrate the efficiency and effectiveness of the novel method.
\end{abstract}

Keywords: along-track interferometric synthetic aperture radar (along-track InSAR); ocean surface current velocity; deep learning; Conditional Generative Adversarial Networks (CGANs)

\section{Introduction}

Ocean surface currents play a key role in momentum, heat, and gas [1], which can affect weather and climate in the relevant sea areas [2-4]. Over the last few decades, several research studies have measured the velocity of ocean surface currents using along-track interferometric (ATI) data [5]. The ATI system is realized by two Synthetic Aperture Radar (SAR) antennas that are some distance apart in the direction of flight and obtain two images of the same scene with a time delay of millisecond magnitude [6,7]. The common alongtrack ATI systems are divided into airborne ATI systems and space-borne ATI systems. The first ATI observations from space were obtained from the Shuttle Radar Topography Mission (SRTM) [5,8,9]. From the theory of along-track InSAR, it can be known that only the velocity of ocean current in the range direction can be measured, which cannot meet the practical requirements. To solve this problem, the dual-beam along-track InSAR system and MA-ATI SAR have been proposed [10-13]. Through the observation in two different directions, two-dimension current velocity can be measured.

The signal processing chain of along-track InSAR data mainly includes two steps. The first step is SAR imaging and along-track interferometric phase extracting, where many uncertainty sources (systematic and random errors, errors caused by the atmospheric phase) may influence the extraction accuracy of the along-track interferometric phase [2]. The second step is the ocean current velocity measurement from the extracted along-track interferometric phase, which is the focus of this paper. Several methods have been proposed 
to calculate the ocean current velocity from along-track interferometric phase. The first method, which we call the direct estimation in this paper, obtains current velocity directly through multiplying the interferometric phase calculated from along-track InSAR data by a linear coefficient. From [14], it is known that the obtained velocity includes the phase velocity of the Bragg wave and the orbital velocity of the large-scale wave; therefore, the velocity estimation error of the direct estimation method is very large in a real-world application. The second method is proposed in [6] to calibrate the surface current field obtained by the along-track interferometric phase via the real current field data of discrete points. However, using the field data to calibrate the surface current field is time consuming and costly in practice. According to the principle that the proportion of the Bragg wave components propagating in different directions of the $\mathrm{L}$ and $\mathrm{C}$ band is approximately equal, the $\mathrm{L}$ and $\mathrm{C}$ dual-band forward orbit interferometric SAR data are proposed to eliminate the phase velocity of the Bragg wave [7]. However, there are few forward orbit interferometric SAR platforms that can receive both $\mathrm{L}$ and $\mathrm{C}$ band data at the same time. The only known aircraft is JPL's AIRSAR, which was decommissioned in 2004. Then, an iterative estimation method is presented by using the established simulation model of along-track interferometric SAR imaging based on the M4S software (the simulation software of ocean surface microwave imaging) [15]. In this method, the optimal matching of the simulated interference phase and the measured interference phase was achieved through iterative correction, and the current field at this time was taken as the optimal solution of the ocean surface current field measured [14-16]. Usually, the iterative estimation method, requiring repeated iterations, is featured by low estimation accuracy. What is worse, the M4S model take a long time to simulate, resulting in low efficiency. In this paper, a novel velocity estimation method of ocean surface currents is proposed based on Conditional Generative Adversarial Networks (CGANs), which can reduce velocity estimation error and improve processing efficiency.

In recent years, with the continuous development of deep learning, this technology has already been used in the field of ocean remote sensing for eddy detection and target recognition $[17,18]$. However, the method of ocean current velocity measurement based on deep learning has not been reported yet. After extensive research of many deep learning network structures, it is found that the Generative Adversarial Networks (GANs) and their improved networks are the most likely to be used for ocean current velocity estimation with along-track InSAR data, which can realize the function of image translation. The GANs $[19,20]$ were proposed by Ian J. Goodfellow in 2014, containing a generative model $\mathrm{G}$ to capture the data distribution and a discriminative model $\mathrm{D}$ to estimate the probability that a sample was produced from the training data rather than $G$ in this network. Since then, many excellent neural network algorithms have emerged, such as the CGANs [21], deep convolutional GANs (DCGANs) [22], and large-scale GANs (BigGANs) [23], which give full play to human creativity to change human life. The greatest merit of the GANs is that they no longer require a hypothetical data distribution, but use a specific distribution to sample directly, so as to obtain data that are infinitely close to the sample in theory. However, the disadvantage of this method is that it is too free since there is no need for premodeling in this method. In the case of larger images with more pixels, the method based on the GANs is difficult to control. As a result, the Conditional Generative Adversarial Networks (CGANs) came into being as an extension of the original GANs. The most widely used CGANs are cycleGAN [24] and pix2pix [25] networks. Mehdi Mirza proposed GANs that are constrained by certain conditions. The conditional variable y is adopted into the modeling of both generator $(G)$ and discriminator $(D)$, while the additional information $y$ is employed to add conditions to the model, so as to guide the process of data generation. This conditional variable y can be developed from a variety of information. The generator uses the U-Net [26] structure, and the discriminator uses the PatchGAN [25] structure. The pix2pix structure draws on the idea of CGANs. When CGANs enter the G network, they will input both the noise and the condition. The fake images generated by the $G$ network will be affected by specific conditions. Therefore, if an image is used as a condition, there 
should a corresponding relationship between the generated fake image and the conditional image, thus realizing an image-to-image translation process [25,27].

In this study, combing with CGANs technology, a novel ocean current velocity estimation method based on the data of along-track InSAR is proposed. By virtue of some image data quality assessment indicators [28], including the root mean squared error (RMSE), correlation coefficient (r), and bias [29], the ocean current velocity estimation results of the newly proposed method are compared and analyzed with those of two commonly used traditional estimation methods (the direct estimation method and the iterative estimation method). The main contributions of this paper are listed as follows in more detail.

1. The deep learning method is applied to the estimation of ocean surface current velocity for the first time. The CGANs extend their retrieval of the current field, with their effectiveness proved. The result of comparing the deep learning method with the traditional methods verifies the superiority of the deep learning method featured by high precision. It is worth noting that the error in velocity measurement is reduced from $0.104 \mathrm{~m} / \mathrm{s}$ (the direct method) and $0.084 \mathrm{~m} / \mathrm{s}$ (the iterative method) to $0.022 \mathrm{~m} / \mathrm{s}$ (the proposed method).

2. After optimization of network parameters, the network with the best ocean surface velocity retrieval effect and the lowest error rate is established, which can be of significant use in optimizing the network design in the future.

3. The generalization ability of the above-mentioned optimal neural network is verified by changing the input interferometric phases under different system parameters, wind field speed, and multi-looks.

\section{Description of the Traditional Methods and the Proposed Method}

In this section, the basic theories of the traditional ocean current velocity estimation methods including the direct method and the iterative method are introduced. Then, the detailed description of the novel method based on CGANs is presented, including the processing flow of the new method, paired datasets for training network, the principle of CGANs, and the network architecture of the new method. Finally, the Image Data Quality (IQA) indexes are presented, which are used in simulation experiments to evaluate the quality of the proposed method and the two conditional methods.

\subsection{The Traditional Methods}

\section{The Direct Method}

The interferometric phase can be used to directly calculate the velocity component of the radar line of sight:

$$
u^{0}=-\frac{\lambda V}{4 \pi B \sin \theta} \varphi^{0},
$$

where $u^{0}$ represents the guessed current field, $\varphi^{0}$ represents the actually obtained alongtrack interference phase, $\lambda$ represents the wavelength of radar, $B$ represents the effective baseline length, $V$ represents the platform velocity, and $\theta$ represents the angle of incident.

2. The Iterative Method

The target velocities obtained by the along-track interferometric phase are Doppler velocities, which are the vector sum of the target radial velocities in all observation areas. In addition to the velocity component of the ocean surface current field, the Doppler velocities also include the velocity resulting from the ocean surface wind, the large-scale wave orbit velocity, and the Bragg wave phase velocity.

$$
u_{\text {Doppler }}=u_{c}+u_{\text {wind }}+u_{o}+u_{b},
$$

where $u_{c}$ represents the surface current field, $u_{\text {wind }}$ represents the velocity produced by the ocean wind, $u_{o}$ represents the large-scale wave orbital velocity, and $u_{b}$ represents the Bragg wave phase velocity. Among them, the sum of the surface velocity caused by the 
ocean surface wind field and the ocean surface current field velocity is considered to be the required surface velocity, that is, $u_{s}=u_{c}+u_{\text {wind }}$.

The iterative algorithm current is shown in Figure 1.

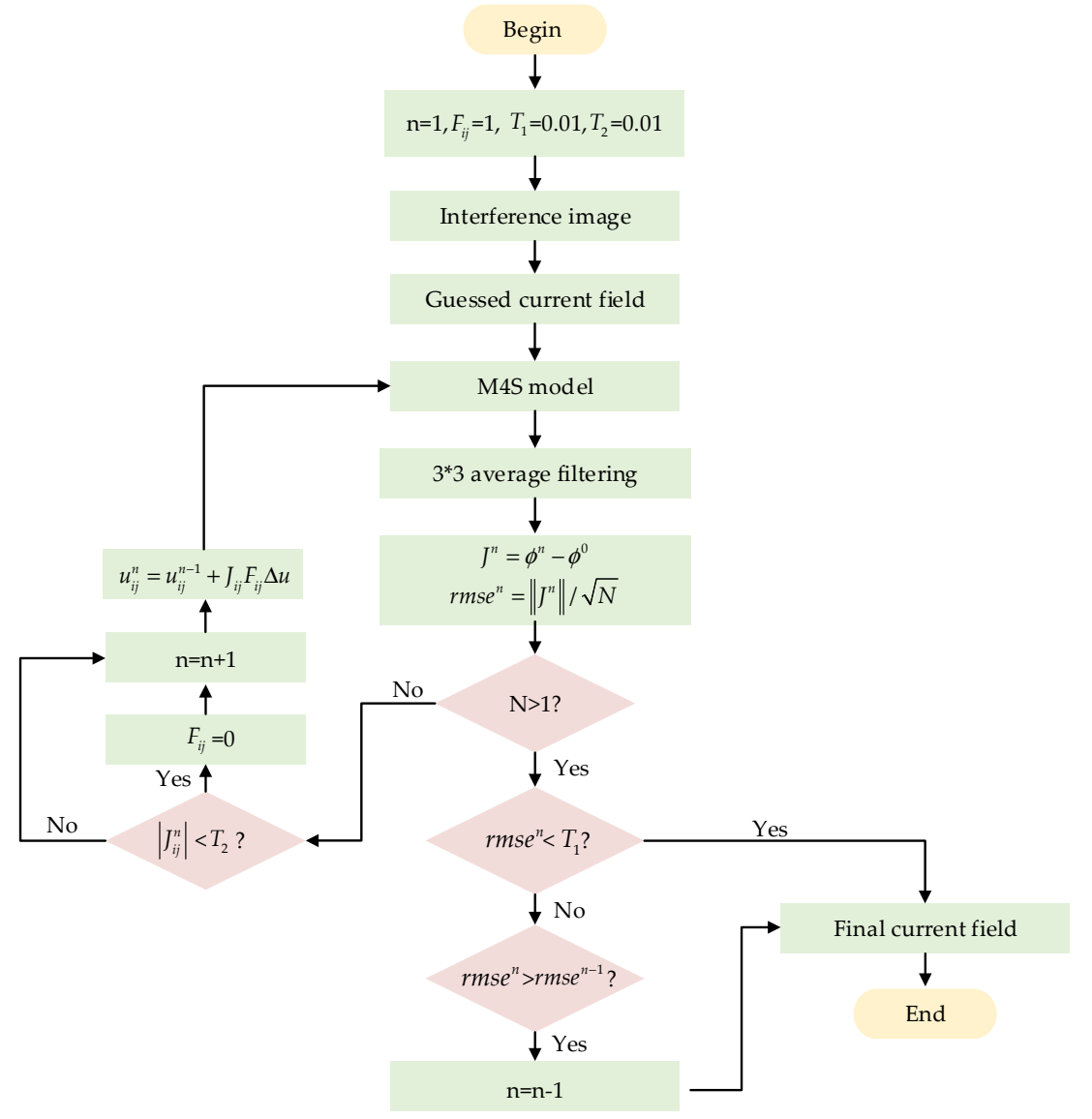

Figure 1. The flowchart of the iterative algorithm.

Specific operation process includes the following steps:

(1) The parameters needed for the correction process of the current field should be initialized. Parameter $\mathrm{N}$ is the number of iterations. Parameter $T_{1}$ is the threshold of root mean square error (RMSE) of the interferometric phase, and the RMSE is used as the error measure in iteration. Parameter $T_{2}$ is the interferometric phase deviation threshold. Parameter $F_{i j}$ is the flow field correction mark.

(2) The calculated surface current field, wind field, simulation system, and other parameters into the M4S software should be input to calculate the actual interferometric phase.

(3) The interferometric phase of the first-guessed current field simulation should be compared with the actual interferometric phase. If the RMSE of the interferometric phase is less than $T_{1}$, this iteration should be stopped. By this time, the current field is the best current field, otherwise, it will display the same result as the last iteration process. The RMSE shall be compared. When it grows larger, the iteration is proved to have diverged, so one should stop the iteration and the output of the current field of the previous iteration. If not, skip to step 4.

(4) A point-wise comparison should be made between the simulated interferometric phase image and the actual interferometric phase image. If the interferometric phase difference at a certain point is less than $T_{1}$, the corresponding current field at that position should not be corrected. If the interferometric phase difference at a certain point is greater than $T_{1}$, the current field should be corrected. Then the corrected surface current field should be input into the M4S software for iterative recalculation. 


\subsection{The New Method Based on CGANs}

\subsubsection{The Flow of the New Method}

Taking the current field and the interferometric phase as input, the CGANs were trained to learn the nonlinear mapping function to obtain the corresponding retrieval current field as the output. The specific method can be divided into three steps (see Figure 2).

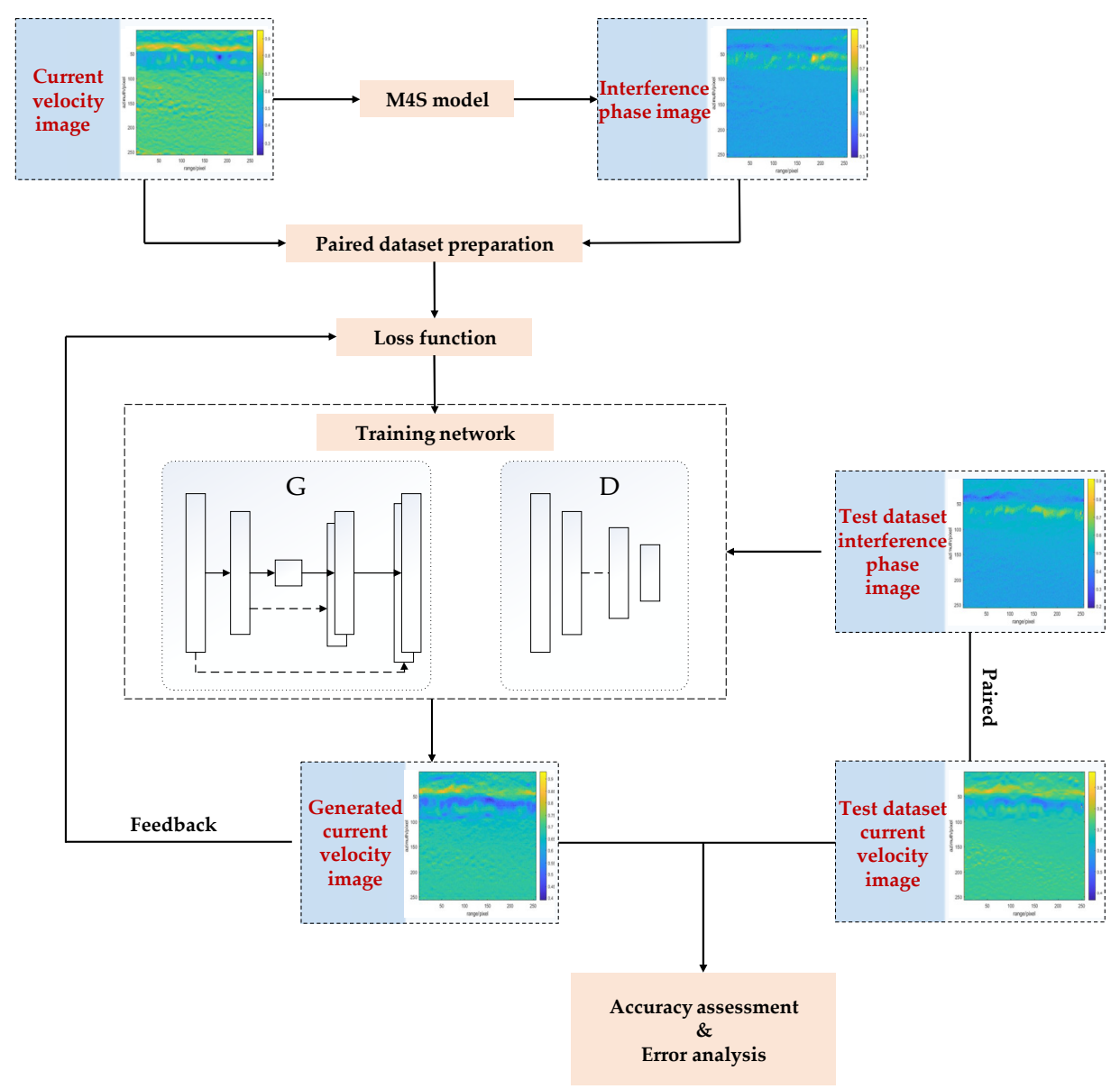

Figure 2. The flowchart of the proposed method.

1. Paired dataset preparation: the current field diagrams and the corresponding interferometric phase diagrams were constructed as the training set to train the pix2pix algorithm.

2. CGANs model training: The estimation model of sea surface current field based on the pix2pix algorithm was constructed. The paired ocean current field diagrams and interferometric phase diagrams were input to train the estimation model until convergence.

3. Accuracy assessment: The interferometric phase diagrams under different parameters in the test dataset were input into the trained current field estimation model to obtain the corresponding ocean surface current field diagrams. Finally, we compared the estimation results and carried out the error analysis to reach some useful conclusions.

\subsubsection{Paired Datasets for Training Network}

M4S is a software toolkit for numerical simulations of the microwave radar imaging of oceanic surface current features and of wind features near the ocean surface, which cause signatures in radar images via hydrodynamic and aerodynamic modulation of the surface wave spectrum. The M4S model mainly includes two calculation modules, namely, the M4Sw (computation of spatially varying wave spectra) and M4Sr (computation of radar signatures). The M4Sw generates the corresponding wave spectrum according to the wave-current interaction model, with the given input of ocean current field and wind field, 
which was established on the basis of the combined ocean surface model. SAR amplitude images and alignment interferometric phases are generated under different radar bands, polarization modes, incident angles, baseline lengths, and other parameters. The principles of the M4S software are shown in Figure 3.

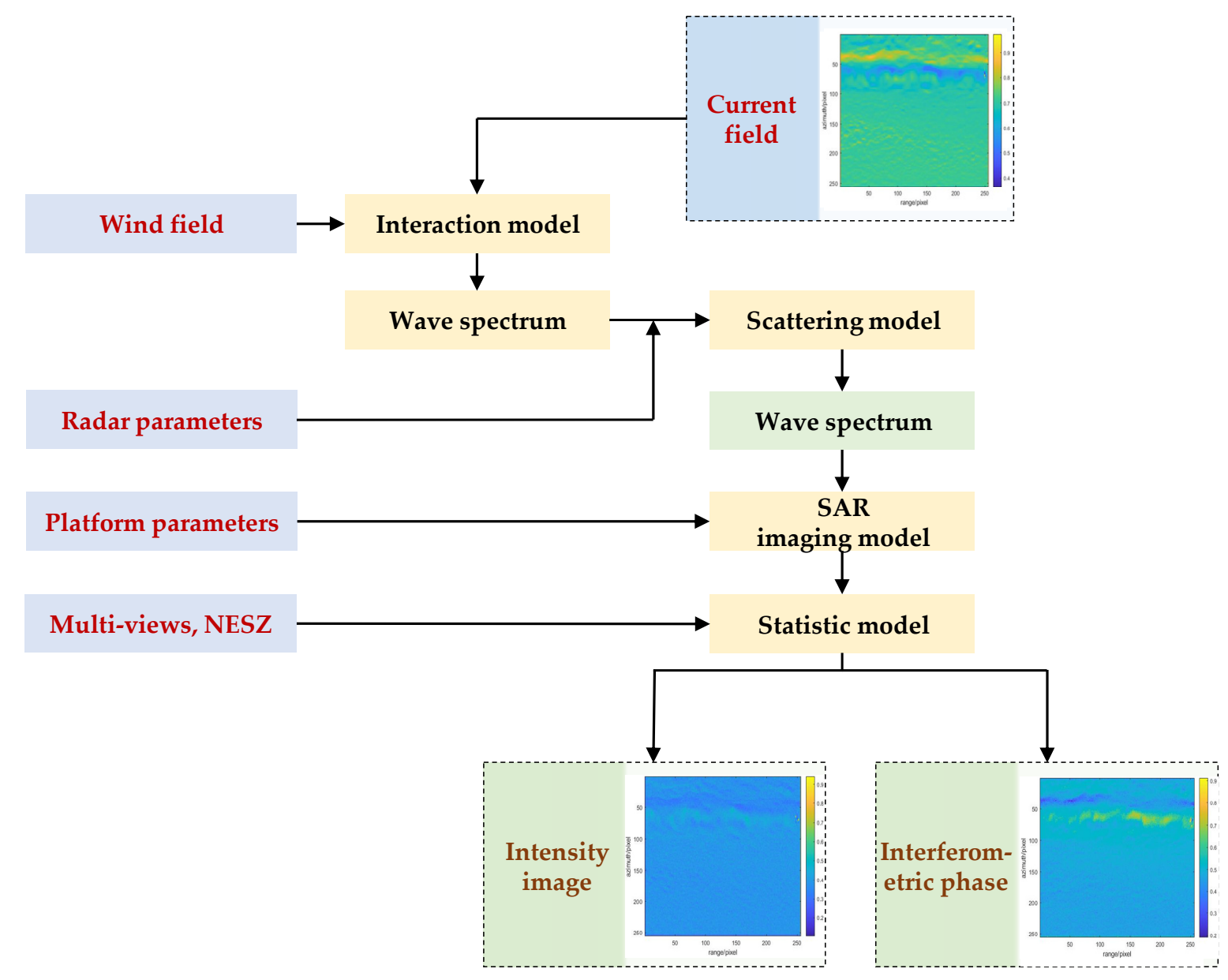

Figure 3. The current chart of interferometric phase generation based on M4S software.

In this paper, the dataset was constructed by the Ocean Surface Current Analyses Real-Time (OSCAR) dataset [30]. The OSCAR data were obtained from JPL Physical Oceanography DAAC and developed by ESR [31]. The horizontal velocity is directly estimated according to the sea surface height, the surface vector wind, and the sea surface temperature, all of which were collected from various satellites and instruments. The network common data form (netCDF) format was adopted for the ocean surface velocity files. Then, we constructed 400 current field maps of $256^{*} 256$ randomly in the various ocean surface velocity files mentioned above. Finally, the 400 current velocity diagrams were input into the M4S model to produce the corresponding interferometric phase diagrams. The current field diagrams and the matching interferometric phase diagrams can be input into the generated confrontation network as the training dataset.

\subsubsection{Conditional Generative Adversarial Networks (CGANs)}

The CGANs are the GANs featured by conditional constraints. In the modeling of both the generation model $(D)$ and the discriminant model $(G)$, the conditional variable $y$ is the input to supplement the model with the additional information $y$, so as to guide the process of data generation. 
The closer the discriminant result of the generated network's data are to 1 , the greater the value of the output $D(G(X))$ of the discriminant network. Therefore, the loss function $L_{G}$ can be expressed as:

$$
L_{G}=\min E_{X}(\ln (1-D(G(X)))) .
$$

The goal of the discriminant network is to make the discriminant result of the real sample close to 1 and the generation data close to 0 , so that both the expectation function $E_{Y, X}(\ln D(Y))$ and the value of $E_{X}(\ln (1-D(G(X))))$ are large enough. Therefore, the loss function $L_{D}$ is expressed as:

$$
L_{D}=\max \left\{E_{Y, X} \ln (D(Y))+E_{X} \ln (1-D(G(X)))\right\} .
$$

Previous approaches have found it beneficial to mix the GAN objective with a more traditional loss, such as $L_{1}$ and $L_{2}$ distance [32]. The $L_{1}$ norm loss function minimizes the sum of the absolute difference between the target value and the estimated value. The $L_{2}$ norm loss function minimizes the sum of squares of the difference between the target value and the estimated value. We selected the $L_{1}$ distance rather than $L_{2}$, as $L_{1}$ encourages less blurring. Meanwhile, the $L_{1}$ distance aims to prevent the model from overfitting for higher accuracy. Therefore, the loss function is expressed as:

$$
L_{1}(G(X))=E_{X, Y}\left(\|Y-G(X)\|_{1}\right) .
$$

By doing so, the whole objective function can be defined as:

$$
G^{*}=\arg \min _{G} \max _{D}\left\{E_{Y, X}(\ln D(Y))+E_{X}(\ln (1-D(G(X))))\right\}+\lambda L_{1}(G(X)),
$$

where $\lambda$ represents the weight parameter.

\subsubsection{Network Architecture}

The model of ocean surface current velocity estimation based on the pix2pix algorithm consists of the generator and the discriminator. Generator $G$, with a U-Net structure, can help share the underlying information between the input and output. The purpose of generator $G$ is to "fool" the discriminator, that is, to hope that the discriminator will regard the fake image as true. The input to the generator is $x$, and the output is its forged image $G(x)$. Discriminator $D$, with a conditional discriminator PatchGan structure, can map the input to the patch $X$ of $N{ }^{*} \mathrm{~N}$, in which the value of $X_{i j}$ represents the probability that all patches are true samples, and the mean value of $X_{i j}$ is the final output of the discriminator. The purpose of discriminant $D$ is to correctly distinguish between real samples and forged samples. When the discriminant is unable to distinguish the sample $G(x)$ generated by the generator from the real sample, the error between the ocean surface current velocity graph obtained and the input velocity graph is small and the accuracy is high, reaching the point of surreal.

The inputs of the generator are the interferometric phases, while the inputs of the discriminator are the current fields and interferometric phases. The outputs of the discriminator are the generated current fields [33] (see Figure 4). 


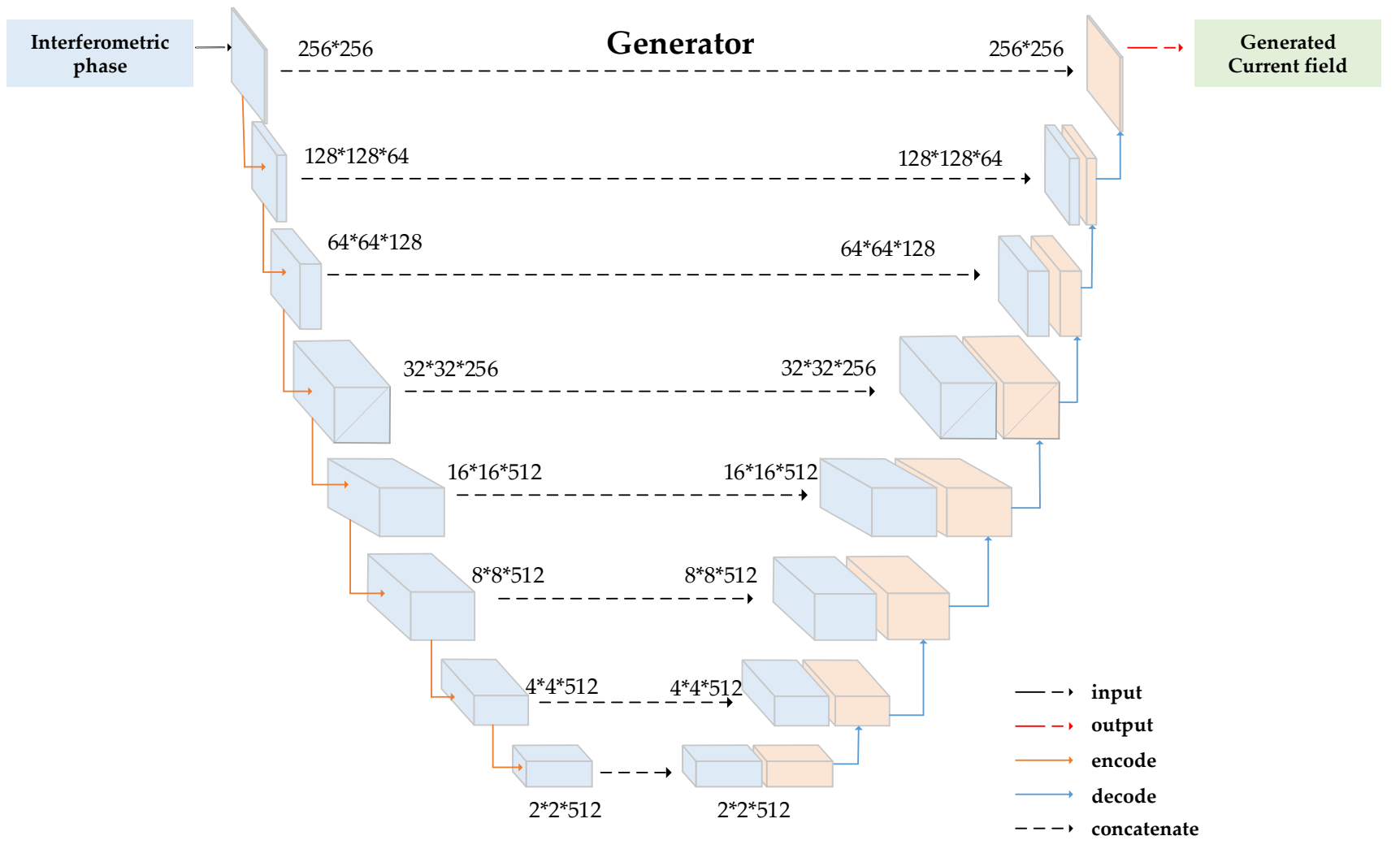

Discriminator

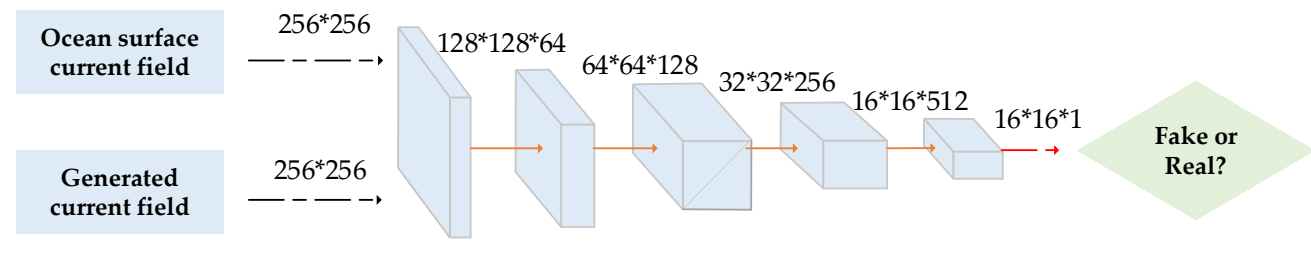

Figure 4. Basic network framework of the pix2pix model.

\section{Experiment}

This section introduces the configuration of the environment and system parameters for the proposed current velocity calculation, the superiority of the method compared with traditional velocity calculation methods, the influence of network structure parameters on the training results, and the generalization ability of the network in detail.

\subsection{Experimental Environment and System Parameters}

Table 1 shows the experimental hardware and software parameters. On this experimental platform, the pix2pix algorithm is used for training and testing in accordance with the algorithm flow in Figure 2. For the parameter selection in the pix2pix algorithm, we adopted the Pytorch framework, selecting U-Net as the backbone feature extraction network and PatchGan as the discriminator, which can recover the low-frequency part of the image well. Both the inputs and outputs in the velocity estimation experiment are images with the dimension of $256 \times 256 \times 3$. Meanwhile, we used the Adam structure as the optimizer to greatly reduce the loss, so as to get a better network structure. The setting of the other super parameters is described in detail in Section 3.4. 
Table 1. Experimental environment.

\begin{tabular}{cccc}
\hline Setting & Software & Setting & Software \\
\hline System & Ubuntu16.04 & Tool & Anaconda3 \\
RAM & 16 GB & Programming & Python3.6 \\
CPU & Intel i5-9600KF 3.7GHz $\times 6$ & IDE & Eclipse \\
GPU & NVIDIA GTX 1080Ti & Framework & Pytorch-GPU \\
Auxiliary tools & MATLAB & Others & CUDA9.0 \\
\hline
\end{tabular}

Table 2 shows the experimental system simulation parameters. The Gaofen-3 satellite is the first civil C-band multipolar SAR satellite in China. The system parameters used in the simulation analysis refer to the relevant parameters of the Gaofen-3 satellite standard band mode, in which the incident angle of alignment interference can be adjusted as required.

Table 2. System simulation parameters.

\begin{tabular}{cccc}
\hline System Parameter & Value & System Parameter & Value \\
\hline Center Frequency & $5.4 \mathrm{GHz}$ & Polarization Mode & VV \\
Effective Baseline & $28 \mathrm{~m}$ & Spatial Resolution & $25 \mathrm{~m}$ \\
Platform Height & $755 \mathrm{~km}$ & Platform Speed & $7000 \mathrm{~m} / \mathrm{s}$ \\
Incident Angle & $35^{\circ}$ & NESZ & $-25 \mathrm{~dB}$ \\
The Number of Multi-Look & 100 & Wind Speed & $5 \mathrm{~m} / \mathrm{s}-15 \mathrm{~m} / \mathrm{s}$ \\
\hline
\end{tabular}

\subsection{Image Data Quality (IQA)}

Reasonable evaluation indicators can help us better evaluate the quality of the generated image. In this paper, in order to quantitatively evaluate the relationship between the ocean surface velocity $m$ obtained through the deep learning training and the input velocity, the following indicators were used for visual comparison: RMSE, $r$, and bias. The mean square error (MSE) is the mean quadratic sum of the difference between the real and predicted values. The RMSE is the square root of the MSE, also known as the fitting standard deviation of the regression system. The smaller the RMSE value is, the higher the image quality and accuracy of the generated image will be.

$$
\operatorname{RMSE}=\sqrt{\frac{1}{m} \sum_{i=1}^{m}\left(y_{i}-\hat{y}_{i}\right)^{2}},
$$

where $m$ represents the observation times, $y_{i}$ represents the real value, and $\hat{y}_{i}$ represents the observed value.

The sum of squares for regression (SSR): the quadratic sum of the difference between the mean values of the predicted data and the original data; the sum of squares for total $(S S T)$ : the quadratic sum of the difference between the original data and the mean values. Meanwhile, $r$, which measures the ratio of SSR and SST, shows the fitting quality through the changes in data. The closer $r$ is to 1 , the better the model fits the data.

$$
r=\sqrt{\frac{S S R}{S S T}} .
$$

Bias measures the difference between the original data and the predicted data. The mean value of $256^{*} 256$ data (the dimension of images in the training dataset is $256^{*} 256$ ) of the input current field matrix and the predicted current field matrix are respectively calculated. The bias represents the difference between these mean values. The closer the bias is to 0 , the higher the quality of the generated image will be. Suitable IQA measurements are conducive to the assessment of translation performance, comparison of translation systems, and visual quality improvement. 


\subsection{Current Velocity Estimation Results Based on the CGANs}

In this experiment, nine different groups of ocean surface current velocity diagrams were applied, as shown in Figure 5. Besides, M4S software was used to generate the corresponding interferometric phase diagrams. In the experiment, nine groups of interferometric phase diagrams are input into the trained neural network to obtain the corresponding estimation of current velocity diagrams, which are shown in Figure 6. In the current field diagram, the resolution in range and azimuth direction is set to $25 \mathrm{~m}$ and the multilook number is set to 10 (range direction) and 10 (azimuth direction). After multi-look processing, the interferometric phase diagram has a resolution of $250 \mathrm{~m}$ in range and azimuth direction.

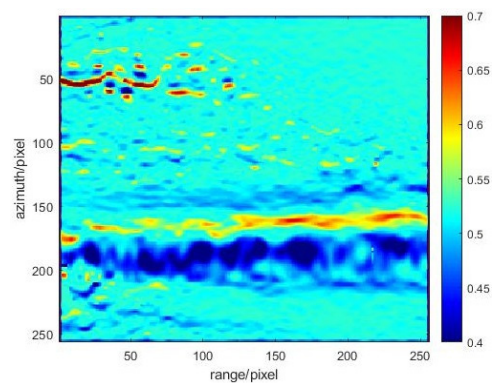

(a)

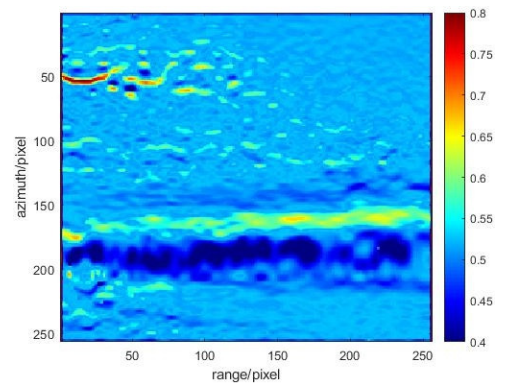

(d)

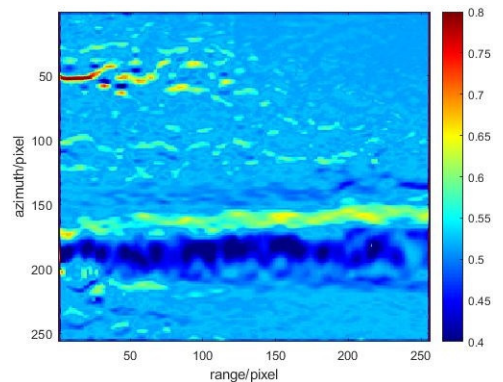

$(\mathrm{g})$

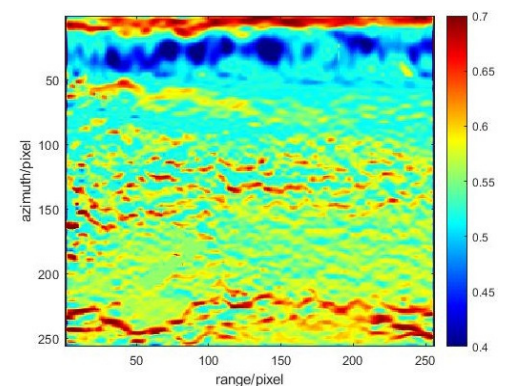

(b)

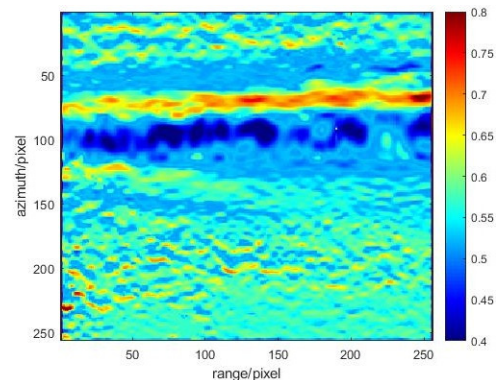

(e)

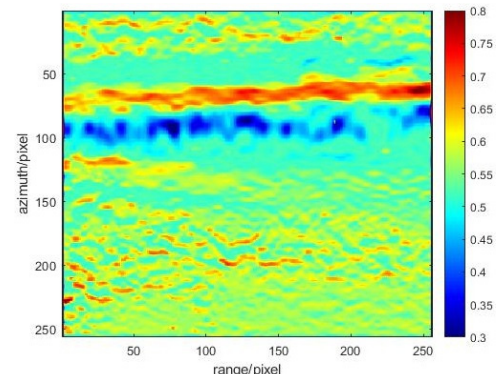

(h)

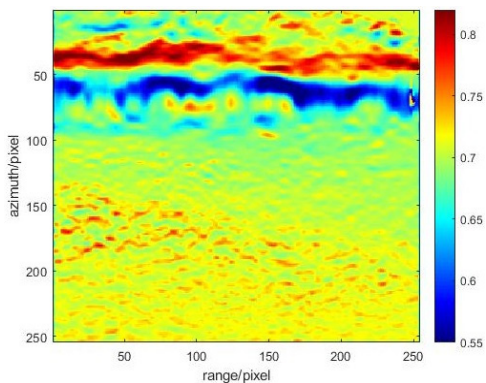

(c)

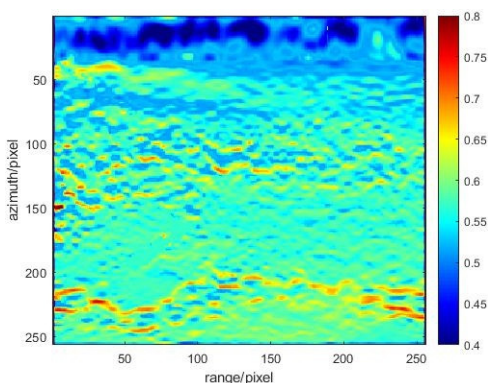

(f)

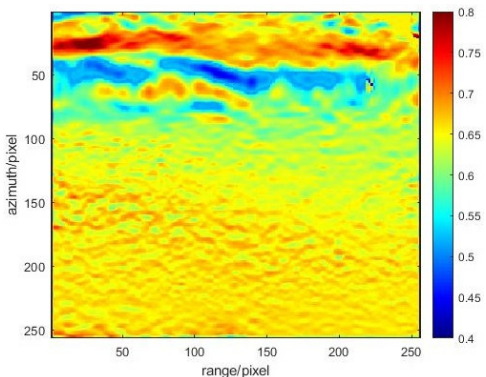

(i)

Figure 5. The input ocean surface current field velocity: (a) sample 1, (b) sample 2, (c) sample 3, (d) sample 4, (e) sample 5, (f) sample 6, (g) sample 7, (h) sample 8, and (i) sample 9. 


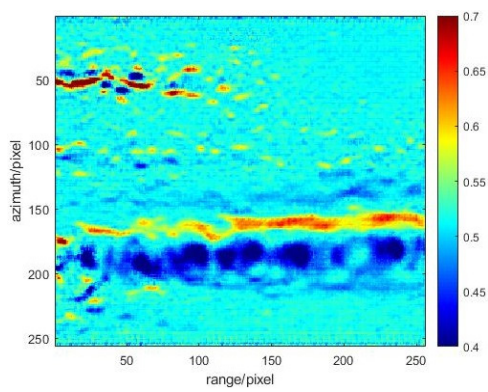

(a)

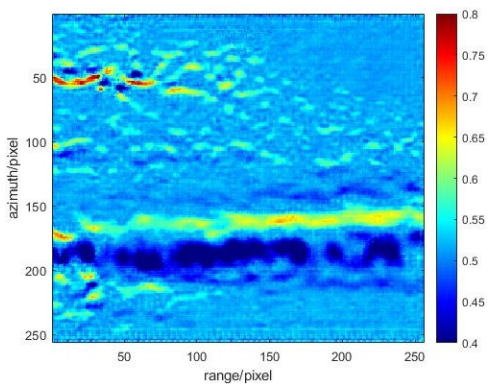

(d)

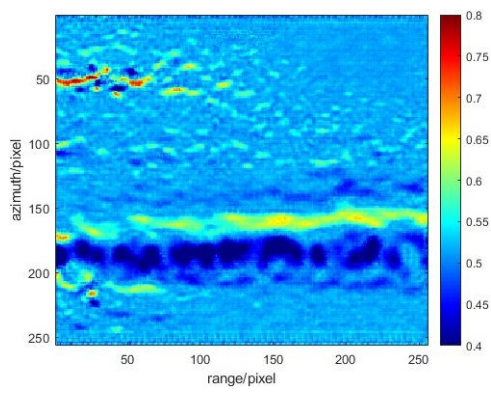

(g)

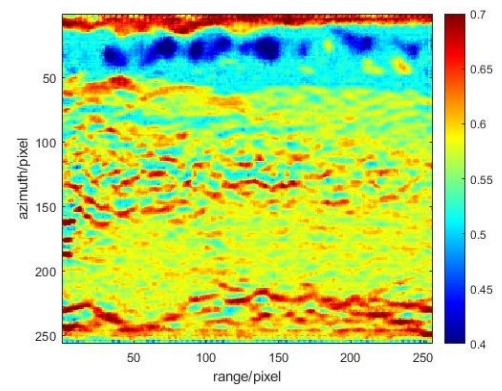

(b)

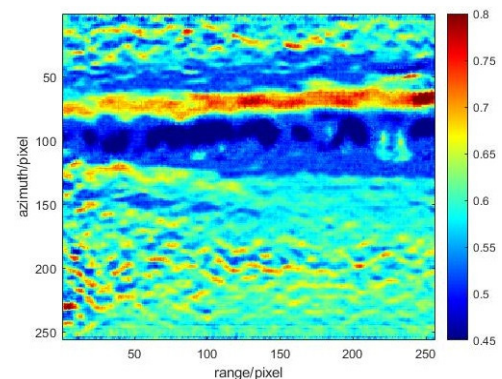

(e)

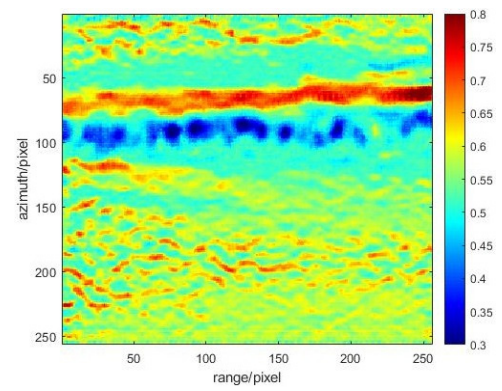

(h)

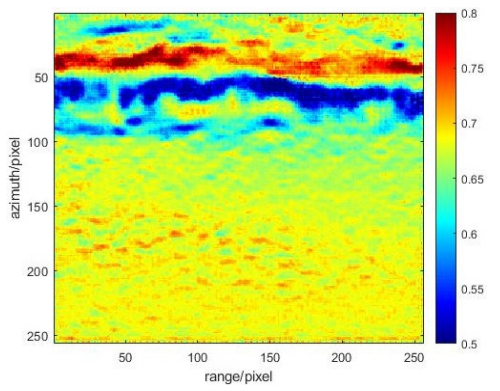

(c)

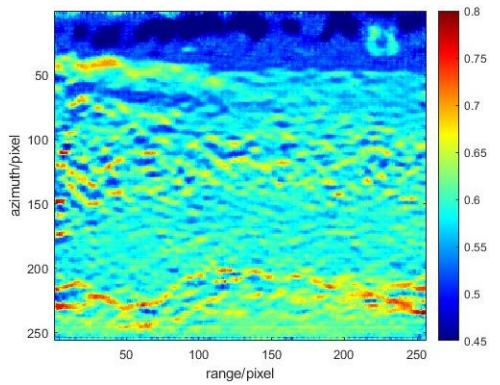

(f)

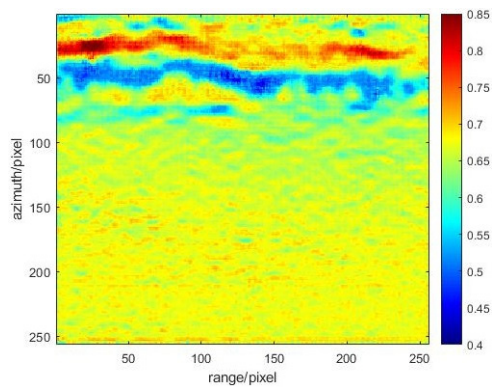

(i)

Figure 6. The estimation ocean surface current field velocity: (a) sample 1, (b) sample 2, (c) sample 3, (d) sample 4, (e) sample 5, (f) sample 6, (g) sample 7, (h) sample 8, and (i) sample 9.

The input current velocity diagram is compared with the current velocity diagram obtained by the neural network test. The IQA consists of RMSE, r, and bias. After listing the IQA indexes of the nine groups of samples, the corresponding three statistical values are calculated. According to the statistical values, it is superior to calculate the ocean surface current velocity by using deep learning which guarantees small error. Table 3 shows the image quality assessment (IQA) results for different inputs.

Table 3. Statistics of the estimation results.

\begin{tabular}{cccc}
\hline & RMSE $(\mathbf{m} / \mathbf{s})$ & $\mathbf{r}$ & Bias $\mathbf{( m / s )}$ \\
\hline Sample 1 & 0.019 & 0.760 & 0.028 \\
Sample 2 & 0.027 & 0.703 & 0.019 \\
Sample 3 & 0.017 & 0.818 & 0.015 \\
Sample 4 & 0.019 & 0.785 & 0.009 \\
Sample 5 & 0.026 & 0.764 & 0.028 \\
Sample 6 & 0.027 & 0.724 & 0.019 \\
Sample 7 & 0.018 & 0.779 & 0.013 \\
Sample 8 & 0.025 & 0.795 & 0.008 \\
Sample 9 & 0.020 & 0.782 & 0.014 \\
Statistical average & 0.022 & 0.768 & 0.017 \\
\hline
\end{tabular}


In Sections 3.4-3.6, the input current velocity diagram shown in Figure 7 is the picture randomly taken from the nine groups of samples in Section 3.3, with the following experiments carried out on the basis of this diagram.

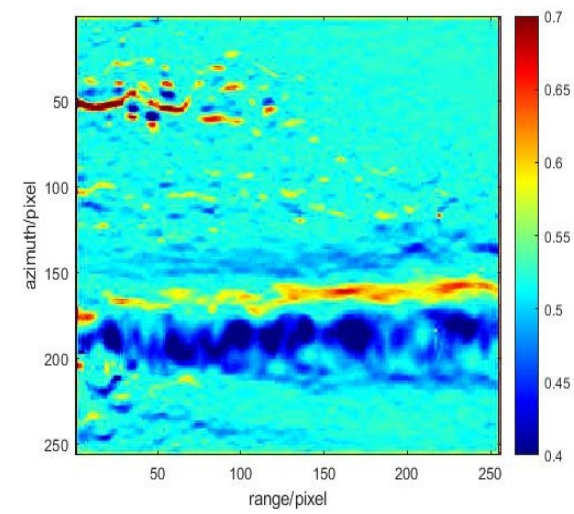

Figure 7. The control group of the input ocean surface current field velocity.

\subsection{Influence of Changing Network Architecture Parameters}

We compared the optimal network of the four hyper-parameters in this experiment. To quantitatively assess the accuracy of the current velocity estimation diagrams, we employed the RMSE, $r$, and bias values in the input current velocity and the estimation of current velocity. To display the results clearly, we employed a scatter diagram to show the density distribution of data using MATLAB. The scatter diagram shows the reflectivity relationship between the generated values and the actual values of the ocean surface current velocity, and the 1:1 line was adopted in the scatter diagram. The closer the points are to the straight line, the more correlated the velocity graph generated by the algorithm is with the input velocity graph, presenting a smaller error and higher accuracy.

(1) Comparison of Different Epochs

It can be seen from Figure 8 and Table 4 that, with other conditions unchanged, the change of epoch has a relatively great impact on the estimation accuracy. When under 300 epochs, the root mean square error is the smallest, with the largest correlation and the smallest bias value between the input current field and the estimation of the current field. In addition, the estimation accuracy is also the highest. The update times of the weights in the neural network increases with the increase with the number of epochs, and the curve also turns from under-fitting to over-fitting. Therefore, in the case of the relatively small datasets, the number of epochs can be increased as many as possible. However, too many epochs will cost too much time, leading to inefficiency. At the same time, the error will be enlarged, which is worthless.

Table 4. Statistics of the estimation results under different epochs.

\begin{tabular}{cccc}
\hline Epoch & RMSE (m/s) & r & Bias (m/s) \\
\hline 100 & 0.022 & 0.670 & 0.015 \\
200 & 0.018 & 0.755 & 0.004 \\
300 & 0.017 & 0.795 & 0.002 \\
400 & 0.019 & 0.763 & 0.005 \\
\hline
\end{tabular}




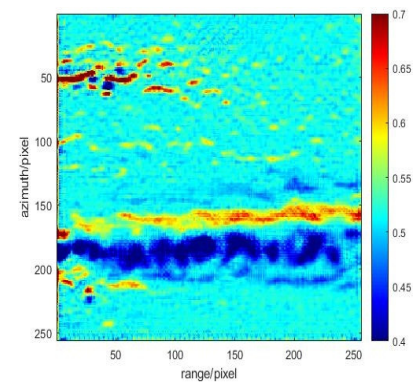

(a)

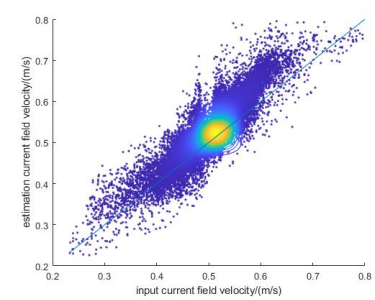

(e)

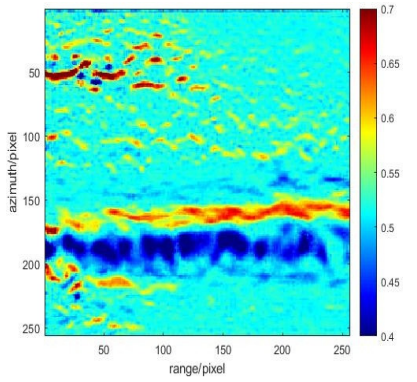

(b)

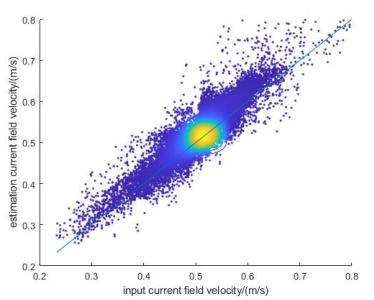

(f)

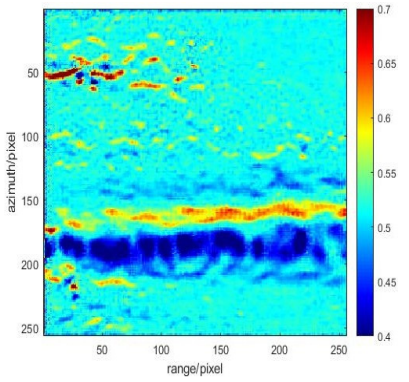

(c)

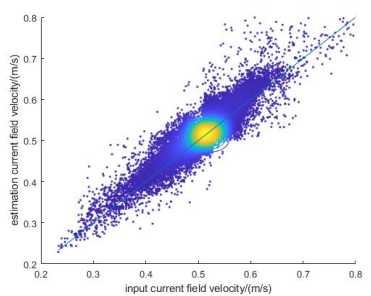

(g)

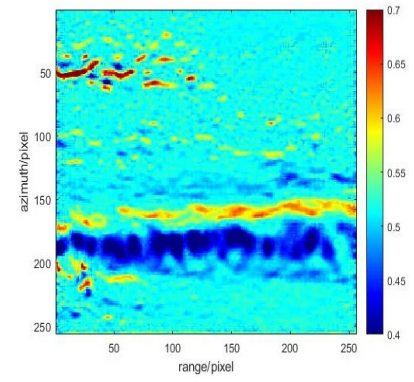

(d)

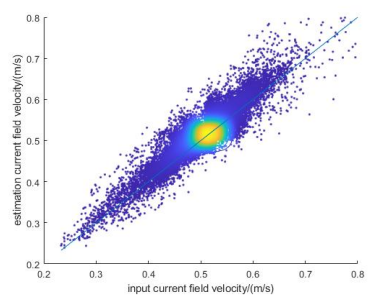

(h)

Figure 8. The estimation velocity of ocean surface currents under the different epochs from (a-d): (a) 100 epochs, (b) 200 epochs, (c) 300 epochs, and (d) 400 epochs. The scatter density map between the estimation velocity of ocean surface currents and the control group velocity of the input ocean surface currents under the different epochs from (e-h):

(e) 100 epochs, (f) 200 epochs, (g) 300 epochs, and (h) 400 epochs.

\section{(2) Comparison of Different Batch Sizes}

It can be seen from Figure 9 and Table 5 that, with other conditions unchanged, the smaller the batch size is, and the better the predicted effect is. When the batch size is 1 , the root mean square error is the smallest, with the largest correlation and the smallest bias value between the input current field and the estimation of the current field. With the growing batch sizes, the generalization ability of the model decreases. Besides, it takes longer to process the same amount of data, requiring more epochs to achieve the same accuracy. On the one hand, the large set of batch size results in fast convergence, less training time, and a stable increase in accuracy. On the other hand, low accuracy affects the factor of randomness.

Table 5. Statistics of the estimation results under different batch sizes.

\begin{tabular}{cccc}
\hline Batch Size & RMSE $(\mathbf{m} / \mathbf{s})$ & $\mathbf{r}$ & Bias $\mathbf{( m / s )}$ \\
\hline 1 & 0.017 & 0.795 & 0.002 \\
2 & 0.025 & 0.736 & 0.009 \\
3 & 0.026 & 0.693 & 0.010 \\
4 & 0.027 & 0.661 & 0.015 \\
\hline
\end{tabular}




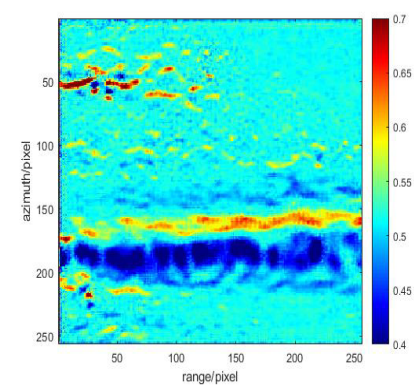

(a)

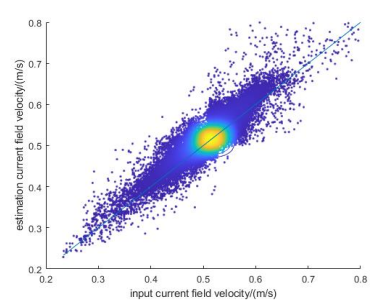

(e)

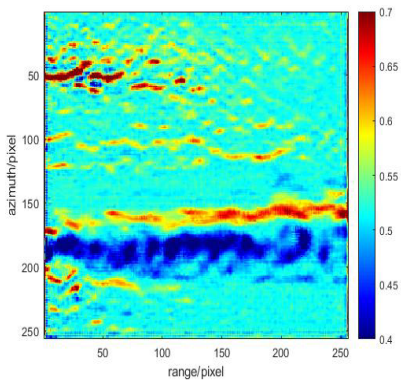

(b)

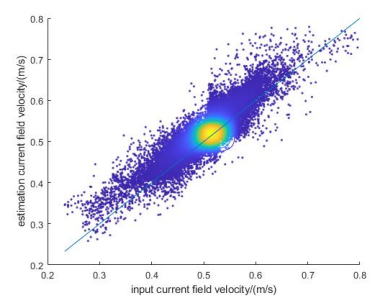

(f)

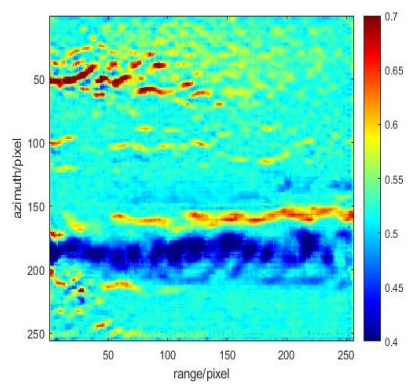

(c)

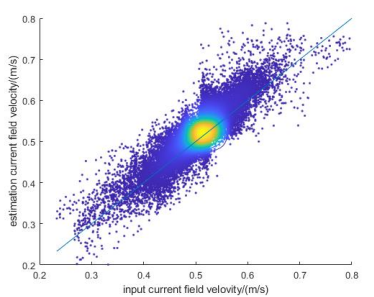

(g)

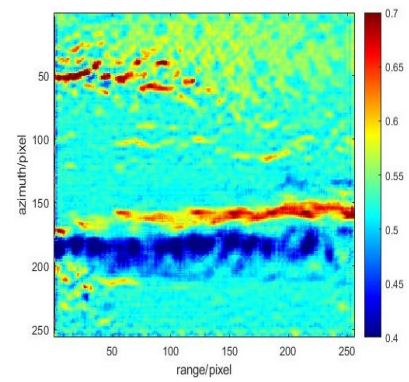

(d)

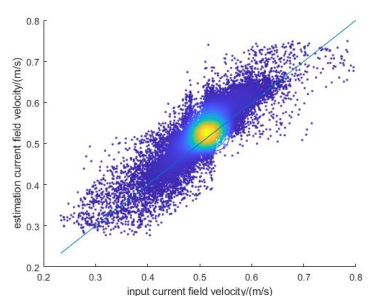

(h)

Figure 9. The estimation velocity of ocean surface currents under the different batch sizes from (a-d): (a) the batch size of 1 , (b) the batch size of 2 , (c) the batch size of 3 , and (d) the batch size of 4 . The scatter density map between the estimation velocity of ocean surface currents and the control group velocity of the input ocean surface currents under the different batch sizes from $(\mathbf{e}-\mathbf{h})$ : (e) the batch size of 1, (f) the batch size of 2, (g) the batch size of 3, and (h) the batch size of 4 .

\section{(3) Comparison of Different Sample Sizes}

According to Figure 10 and Table 6, it can be found that with other conditions unchanged, the predicted effect will improve with the increasing number of samples in the training set. When there are 400 samples, the root mean square error is the smallest, with the largest correlation and the smallest bias value between the input current field and the estimation of the current field. However, it takes a lot of time to obtain the corresponding interferometric phase diagram through the M4S software. Even worse, the ocean surface current velocity diagram is difficult to get, apart from difficulties in the construction of the dataset. Therefore, the dataset can be expanded through certain image enhancement methods, such as clipping, translation, rotation, brightness, saturation, etc. The richer the dataset is, the better the velocity graph obtained by deep learning is, and the smaller the error is.

Table 6. Statistics of the estimation results under different sample numbers.

\begin{tabular}{cccc}
\hline $\begin{array}{c}\text { The Number of } \\
\text { Samples }\end{array}$ & RMSE (m/s) & r & Bias (m/s) \\
\hline 100 & 0.024 & 0.652 & 0.017 \\
200 & 0.023 & 0.664 & 0.014 \\
300 & 0.021 & 0.670 & 0.009 \\
400 & 0.017 & 0.795 & 0.002 \\
\hline
\end{tabular}




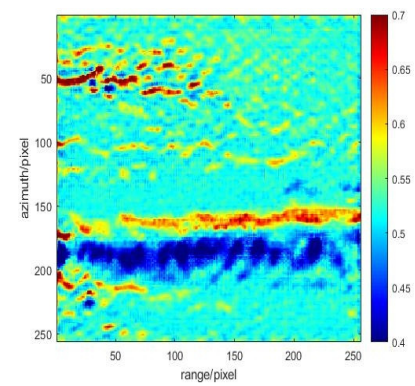

(a)

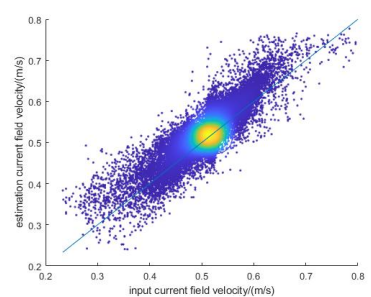

(e)

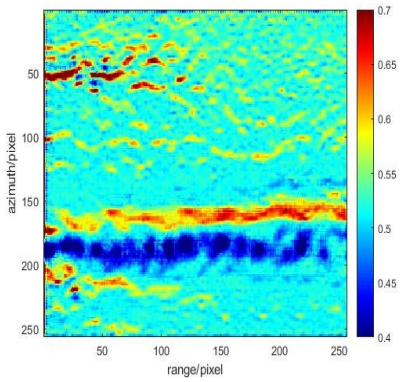

(b)

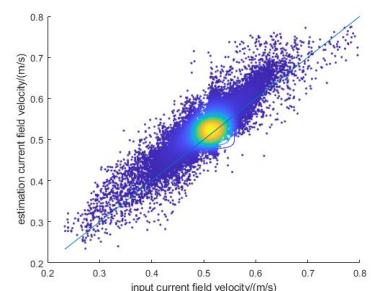

(f)

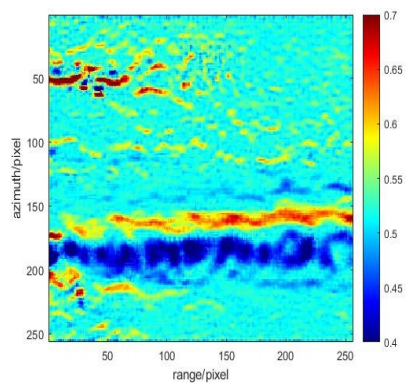

(c)

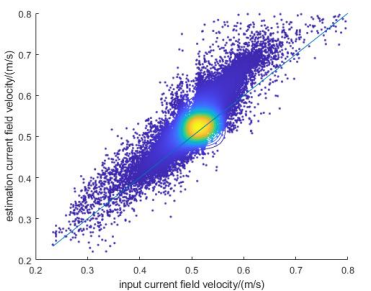

(g)

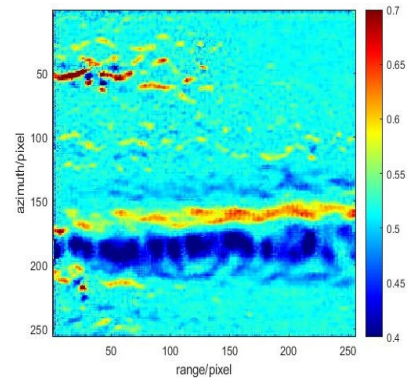

(d)

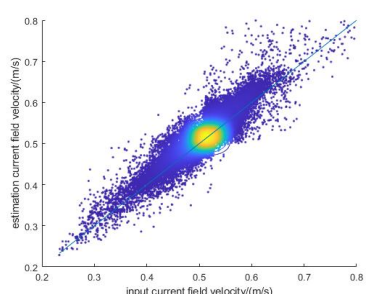

(h)

Figure 10. The estimation velocity of ocean surface currents under the different sample sizes from (a-d): (a) 100 samples, (b) 200 samples, (c) 300 samples, and (d) 400 samples. The scatter density map between the estimation velocity of the ocean surface currents and the control group velocity of the input ocean surface currents under the different sample sizes from (e-h): (e) 100 samples, (f) 200 samples, (g) 300 samples, and (h) 400 samples.

\section{(4) Comparison of Different Learning Rates}

In order to give full play to the gradient descent method, the value of the learning rate should be set within an appropriate range. The learning rate determines how fast the parameters move to the optimal value. If the learning rate is set too low, the convergence will be accelerated greatly with more training time. An excessive learning rate may cause the parameters to oscillate back and forth between the optimal solution on both sides. The algorithm fails to converge for a long time. It can be seen from Figure 11 and Table 7 that with other conditions unchanged, the initial learning rate is not very high. Besides, when the initial learning rate is 0.0002 , the root mean square error is the smallest, with the largest correlation and the smallest bias value between the input current field and the estimation of the current field, thus indicating the highest estimation accuracy.

Table 7. Statistics of the estimation results under different learning rates.

\begin{tabular}{cccc}
\hline Learning Rate & RMSE $(\mathbf{m} / \mathbf{s})$ & $\mathbf{r}$ & Bias $(\mathbf{m} / \mathbf{s})$ \\
\hline 0.0001 & 0.022 & 0.700 & 0.008 \\
0.0002 & 0.017 & 0.800 & 0.002 \\
0.0003 & 0.021 & 0.736 & 0.006 \\
0.0004 & 0.022 & 0.728 & 0.007 \\
\hline
\end{tabular}




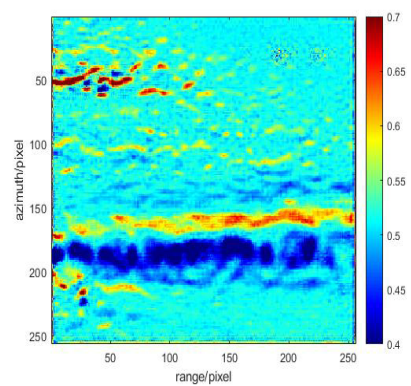

(a)

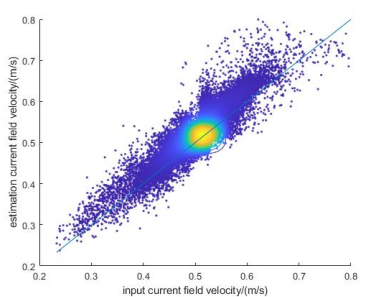

(e)

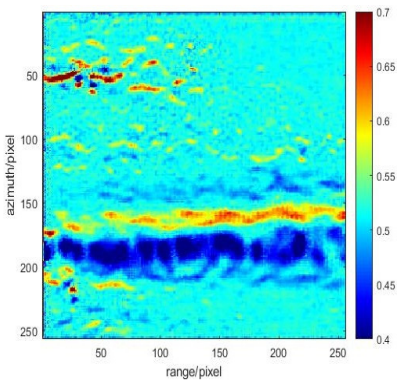

(b)

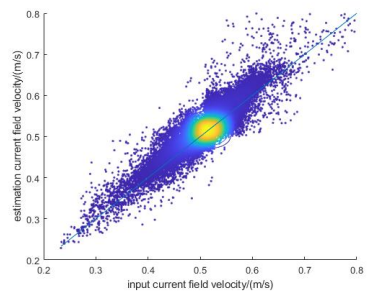

(f)

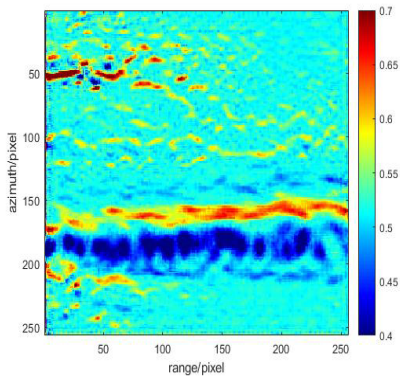

(c)

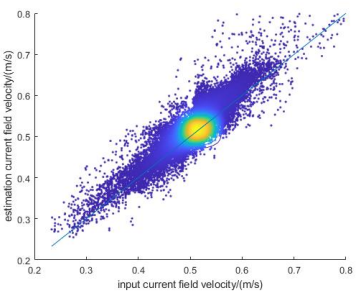

(g)

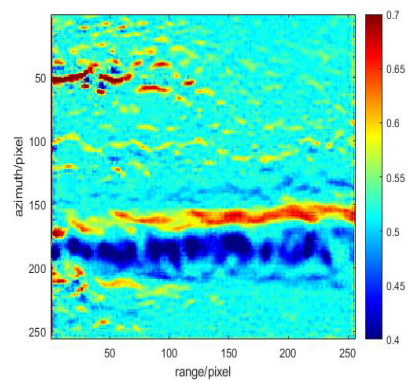

(d)

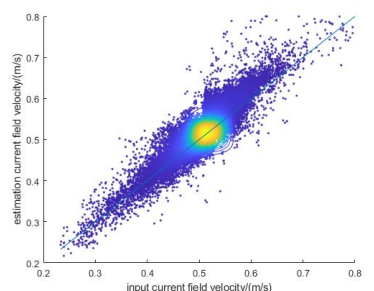

(h)

Figure 11. The estimation velocity of ocean surface currents under the different learning rates from (a-d): (a) 0.0001, (b) 0.0002 , (c) 0.0003 , and (d) 0.0004 . The scatter density map between the estimation velocity of the ocean surface currents and the control group velocity of the input ocean surface currents under the different learning rates from (e-h): (e) 0.0001 , (f) $0.0002,($ g) 0.0003 , and (h) 0.0004 .

\subsection{Comparison of Different Methods Based on the Simulated Data}

According to the result specified in Section 3.4, when the hyper-parameters of the training network are under 300 epochs and 400 samples, with the batch size of 1 and the initial learning rate at 0.0002 , the test result is obviously better than those of the networks using other hyper-parameters. Compared with the direct estimation method and the iterative method, the machine learning method using the above-mentioned hyperparameters is obviously preferred, featured by minimum error and high efficiency in the calculation of the current field velocity. The comparison results of the traditional methods and the new method can be seen from Figure 12, Figure 13, Figure 14 and Table 8.

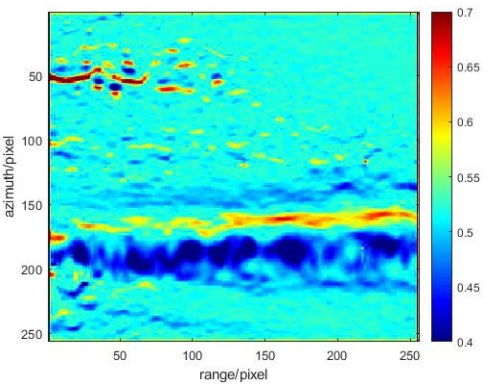

(a)

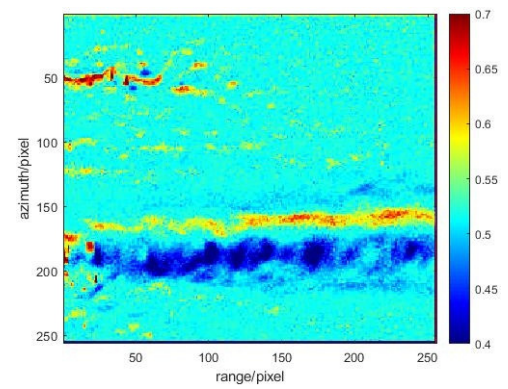

(b)

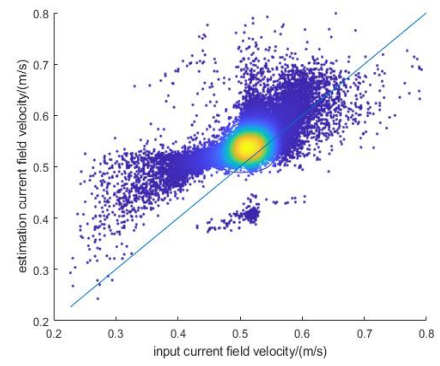

(c)

Figure 12. The velocity calculation results based on the direct method: (a) the input velocity, (b) the current velocity calculated directly from the interferometric phase, and (c) the scatter density map of the input and calculated current velocities. 


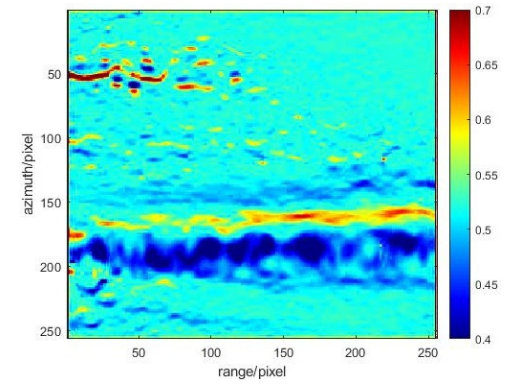

(a)

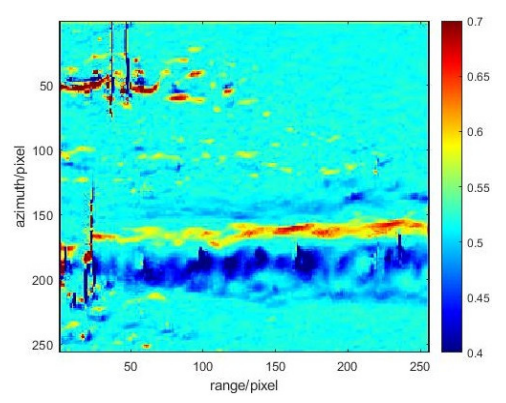

(b)

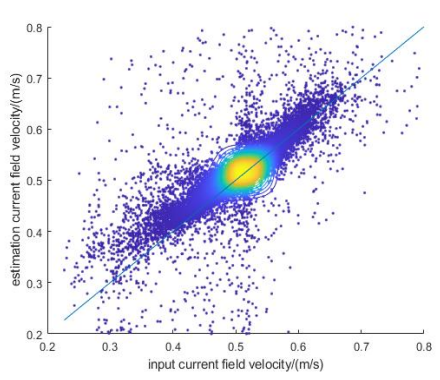

(c)

Figure 13. The velocity calculation results based on the iterative method: (a) the input velocity, (b) the iterative current velocity, and (c) the scatter density map of the input and calculated current velocities.

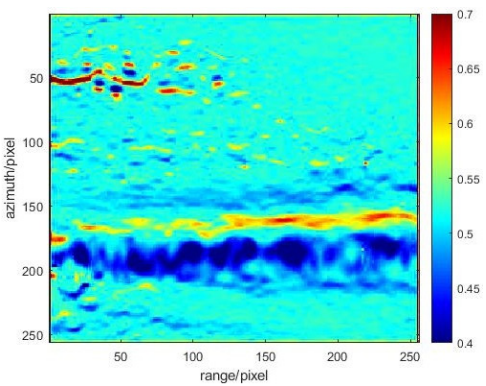

(a)

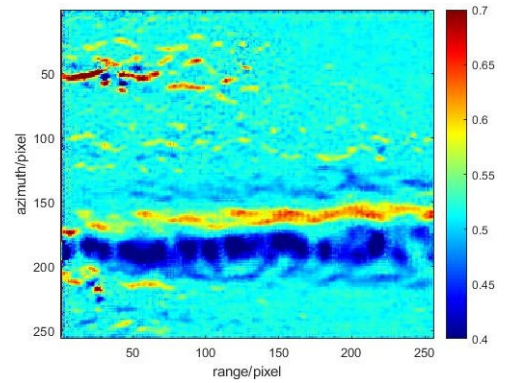

(b)

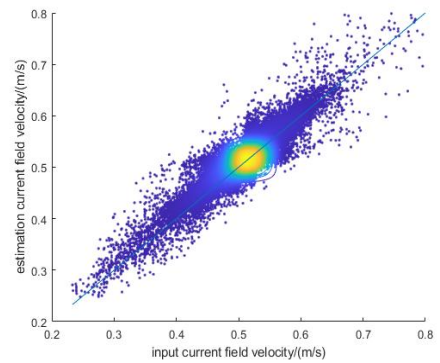

(c)

Figure 14. The velocity calculation results based on the proposed method: (a) the input velocity, (b) the calculated velocity obtained through the trained pix2pix network, and (c) the scatter density map of the input and calculated current velocities.

Table 8. Statistics of the estimation results under different methods.

\begin{tabular}{cccc}
\hline Method & RMSE $(\mathbf{m} / \mathbf{s})$ & $\mathbf{r}$ & Bias $(\mathbf{m} / \mathbf{s})$ \\
\hline Direct Method & 0.104 & 0.714 & 0.028 \\
Iterative Method & 0.084 & 0.641 & 0.010 \\
Proposed Method & 0.017 & 0.795 & 0.002 \\
\hline
\end{tabular}

According to the table and Equation (7) discussed above, it is found that:

$$
u^{0}=-1.9269 \varphi^{0}
$$

The comparison results of the traditional methods and the new method can be seen from Figure 12, Figure 13, Figure 14 and Table 8.

Figure $12 \mathrm{~b}$, as a result of transformation from the interferometric phase diagram to the horizontal line-of-sight velocity component is also the initial guessed current field of velocity retrieval through the iterative method. It is obvious that the RMSE and bias are the worst among the three methods in terms of their values. This is because the velocity diagram of the direct method is obtained directly by multiplying the interferometric phase data by a coefficient, so that there is a large amount of noise interference in the direct velocity retrieval diagram. According to the mainstream iterative method to calculate the sea surface velocity algorithm [14], it is known that the RMSE between the predicted current field and the input current field is about $0.05 \mathrm{~m} / \mathrm{s}$, but the iteration error we measured was slightly greater than $0.05 \mathrm{~m} / \mathrm{s}$ in this paper. The retrieval error mainly results from insufficient filtering, multi-look, and other processing of the image edge. Consequently, it can be hard to get rid of the edge noise. Obviously, the RMSE of the velocity calculation method based on deep learning proposed in this paper is around $0.02 \mathrm{~m} / \mathrm{s}$, leading to 
higher accuracy in retrieval. Meanwhile, $r$ is the highest and bias is the lowest among the three methods, which also proves the superiority of the proposed method.

\subsection{Influence of Changing Parameters}

Based on the above optimal network structure, this section changed the three parameters of wind speed, multi-look, and incident angle to verify the generalization ability of the network.

\section{(1) Comparison of Different Wind Velocities}

According to Figure 15 and Table 9, it is discovered that with other conditions unchanged, the change of the wind field speed can impact the estimation accuracy significantly. When the wind field speed of the test dataset is consistent with that of the training set, the input current field is consistent with the estimation of the current field. Given the smallest root mean square error, the estimation accuracy is the highest. Nevertheless, the greater the wind speed is, the greater the error is.

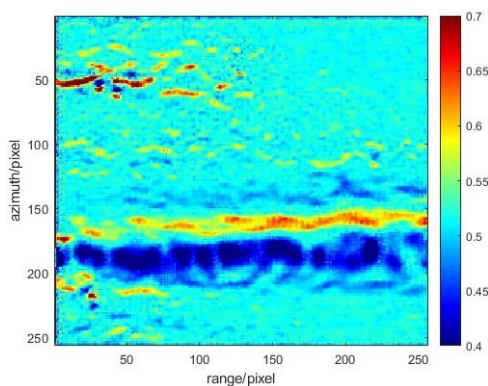

(a)

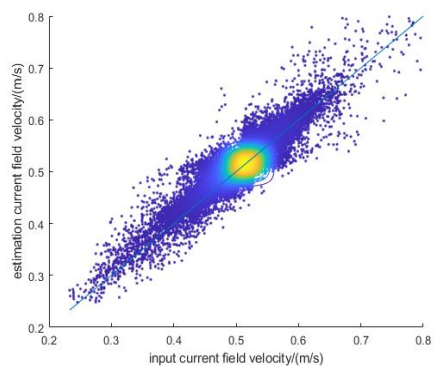

(d)

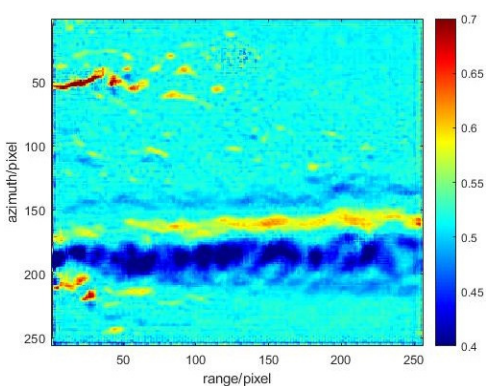

(b)

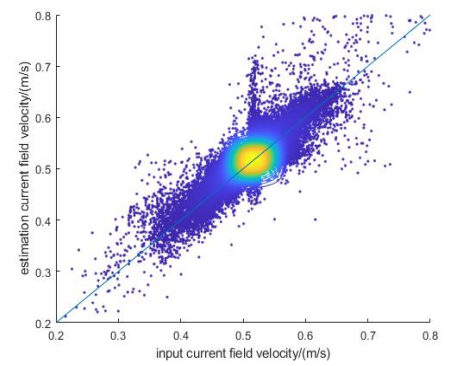

(e)

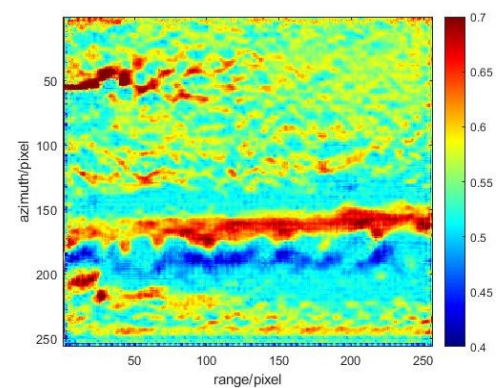

(c)

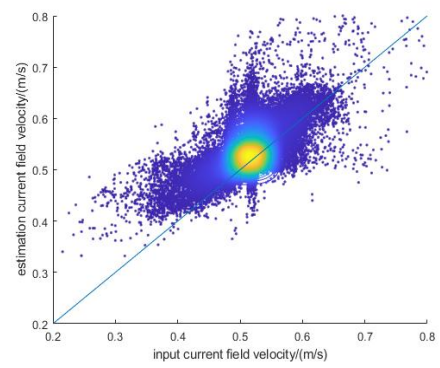

(f)

Figure 15. The estimation velocity of ocean surface currents under the different wind velocities from (a-c): (a) $5 \mathrm{~m} / \mathrm{s}$, (b) $10 \mathrm{~m} / \mathrm{s}$, and (c) $15 \mathrm{~m} / \mathrm{s}$. The scatter density map between the estimation velocity of ocean surface currents and the control group velocity of the input ocean surface currents under the different wind velocities (d-f): (d) $5 \mathrm{~m} / \mathrm{s},(\mathbf{e}) 10 \mathrm{~m} / \mathrm{s}$, and (f) $15 \mathrm{~m} / \mathrm{s}$.

Table 9. Statistics of the estimation results under different wind velocities.

\begin{tabular}{cccc}
\hline Wind Velocity (m/s) & RMSE $(\mathbf{m} / \mathbf{s})$ & $\mathbf{r}$ & Bias $(\mathbf{m} / \mathbf{s})$ \\
\hline 5 & 0.017 & 0.795 & 0.002 \\
10 & 0.020 & 0.701 & 0.003 \\
15 & 0.030 & 0.373 & 0.019 \\
\hline
\end{tabular}

\section{(2) Comparison of Different Multi-Looks}

From Figure 16 and Table 10, we can see that with other conditions unchanged, the impact of multi-looks on the estimation accuracy is limited. Regardless of changes in looks, the RMSE between the input current field velocity and the estimation of current field velocity hardly changes. 


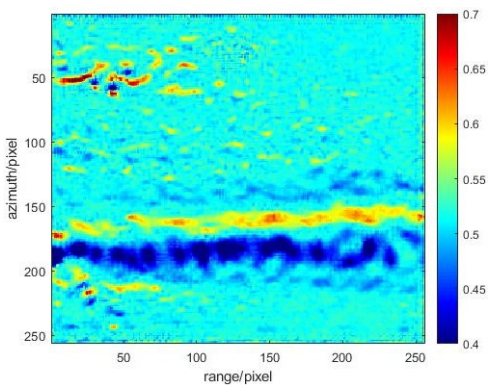

(a)

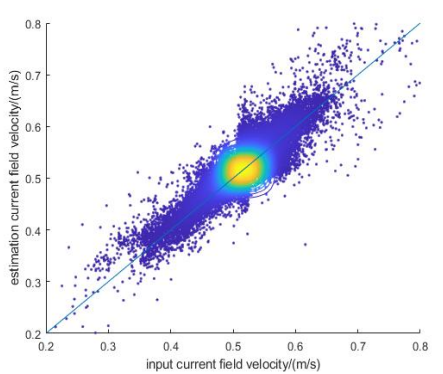

(d)

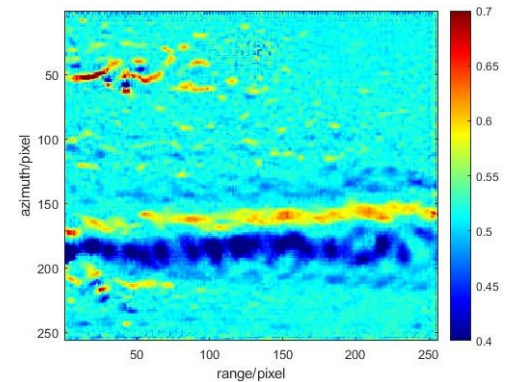

(b)

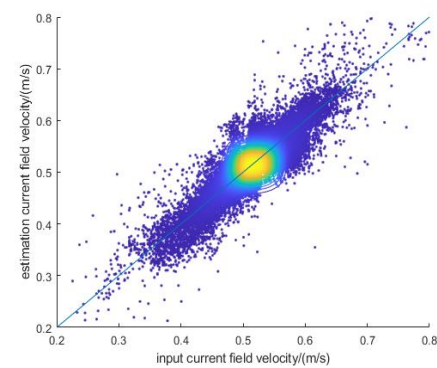

(e)

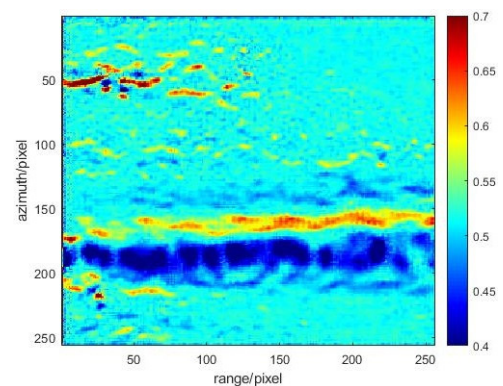

(c)

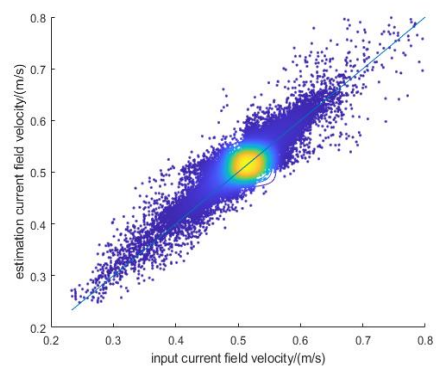

(f)

Figure 16. The estimation velocity of ocean surface currents under the different multi-looks from (a-c): (a) 16 looks, (b) 64 looks, and (c) 100 looks. The scatter density map between the estimation velocity of ocean surface currents and the control group velocity of the input ocean surface currents under the different multi-looks from (d-f): (d) 16 looks, (e) 64 looks, and (f) 100 looks.

Table 10. Statistics of the estimation results under different multi-looks.

\begin{tabular}{cccc}
\hline Multi-Look & RMSE (m/s) & $\mathbf{r}$ & Bias $(\mathbf{m} / \mathbf{s})$ \\
\hline 16 & 0.020 & 0.728 & 0.002 \\
64 & 0.019 & 0.750 & 0.002 \\
100 & 0.017 & 0.795 & 0.002 \\
\hline
\end{tabular}

\section{(3) Comparison of Different Incident Angles}

Figure 17 and Table 11 indicate that, with other conditions unchanged, changes in the incident angle have a relatively large influence on the estimation accuracy. When the incident angle equals that of the training set, which is $35^{\circ}$, the root mean square error between the input current field velocity and the estimation of current field velocity is the smallest with the highest estimation accuracy.

Table 11. Statistics of the estimation results under different incident angles.

\begin{tabular}{cccc}
\hline Incident Angle $\left.\mathbf{(}^{\circ}\right)$ & RMSE (m/s) & $\mathbf{r}$ & Bias $(\mathbf{m} / \mathbf{s})$ \\
\hline 20 & 0.031 & 0.296 & 0.016 \\
28 & 0.020 & 0.704 & 0.003 \\
35 & 0.017 & 0.795 & 0.002 \\
43 & 0.018 & 0.761 & 0.005 \\
50 & 0.026 & 0.508 & 0.014 \\
\hline
\end{tabular}




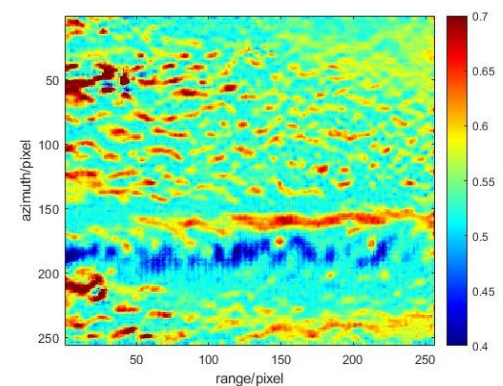

(a)

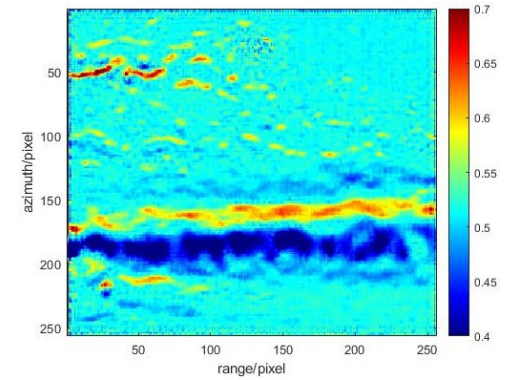

(d)

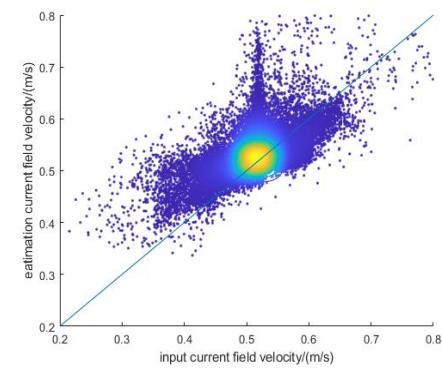

$(\mathbf{f})$

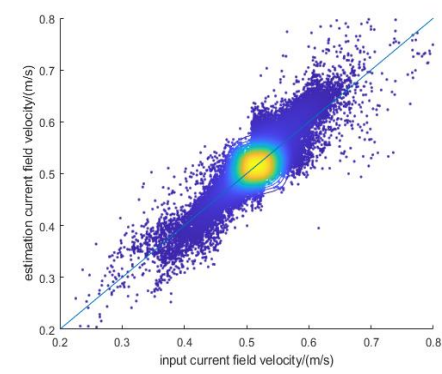

(i)

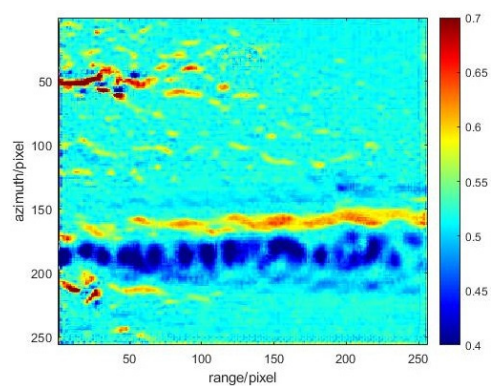

(b)

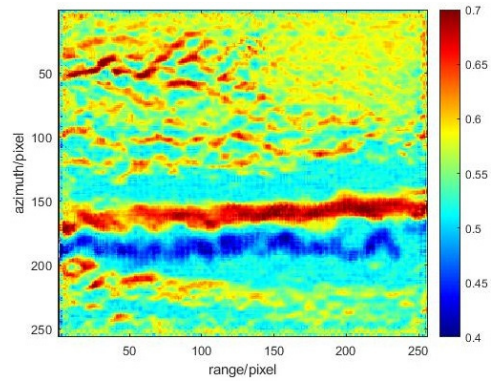

(e)

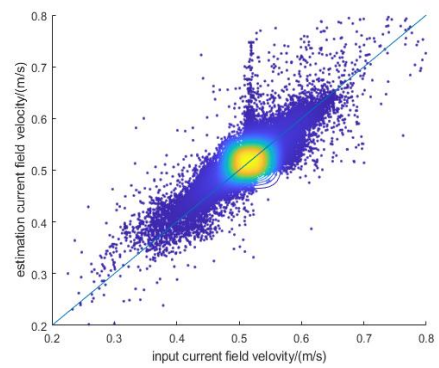

(g)

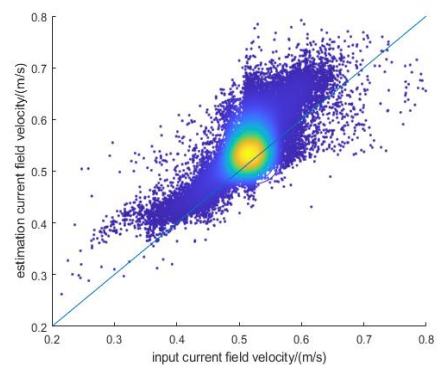

(j)

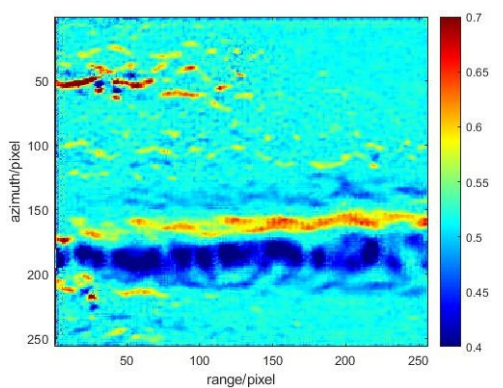

(c)

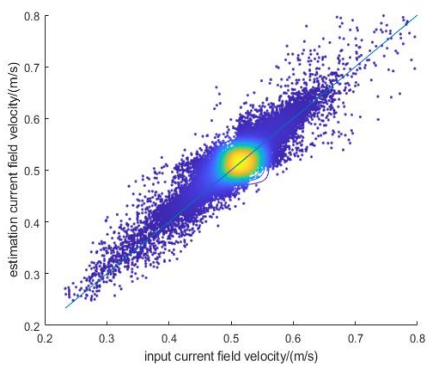

(h)

Figure 17. The estimation velocity of ocean surface currents under the different incident angles from (a-e): (a) $13^{\circ},(\mathbf{b}) 28^{\circ}$, (c) $35^{\circ}$, (d) $43^{\circ}$, and (e) $50^{\circ}$. The scatter density map between the estimation velocity of ocean surface currents and the control group velocity of the input ocean surface currents under the different incident angles from (f-j): (f) $13^{\circ},(\mathrm{g}) 28^{\circ}$, (h) $35^{\circ}$, (i) $43^{\circ}$, and (j) $50^{\circ}$.

\subsection{Verification of the Real Data}

To verify the effectiveness of the proposed algorithm, real data of the two-channel along-track InSAR mode from the Gaofen-3 spaceborne SAR system are processed. It should be pointed out that the ocean current velocity measurement mode is not designed for Gaofen-3 spaceborne SAR system; therefore, the corresponding cooperation experiments are not carried out. The basic SAR processing and interferogram generation were performed at the Aerospace Information Research Institute, Chinese Academy of Sciences. Figure 18 
shows the real data after SAR imaging with the size of 13182(azimuth)*15316(range). In Figure 18, the sea surface area of 8192 (azimuth)*8192(range) is extracted for the real data verification, which is marked with red rectangle. According to local weather records, the wind speed is around $4.5 \mathrm{~m} / \mathrm{s}$ and the wind direction is about $130^{\circ}$ along the SAR azimuth direction. After the flat-earth phase removal and phase calibration, the interferometric phase of the extracted area is shown in Figure 19, and no useful information can be found due to the relatively low SNR. Therefore, 1024 multi-looks and three times $10^{*} 10$ element boxcar averaging are performed, and the result is shown in Figure 20 with the size of 256(azimuth)*256(range). From Figure 20, it can be found that the texture structure of the extracted interferometric phase is in accordance with the texture structure of extracted SAR image in Figure 18. Due to the lack of real current field data of the extracted area, in the following experiment, the three different algorithms are compared based on the extracted interferometric phase in Figures 21-23.

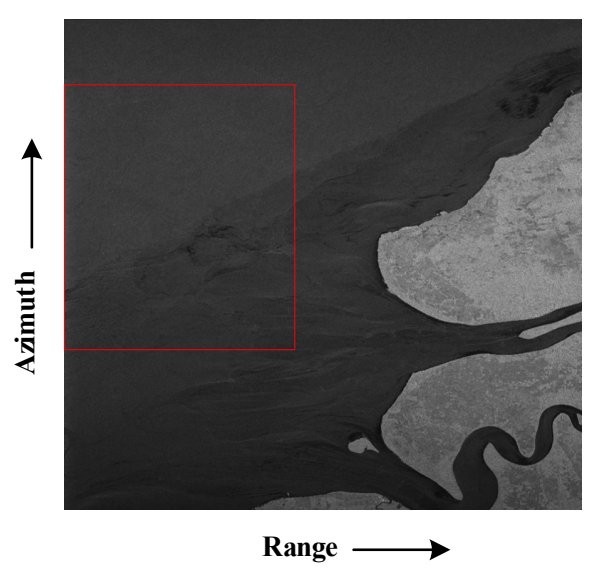

Figure 18. Real data from the two-channel along-track InSAR mode of the Gaofen-3 spaceborne SAR system (size: 13182(azimuth)*15316(range)).

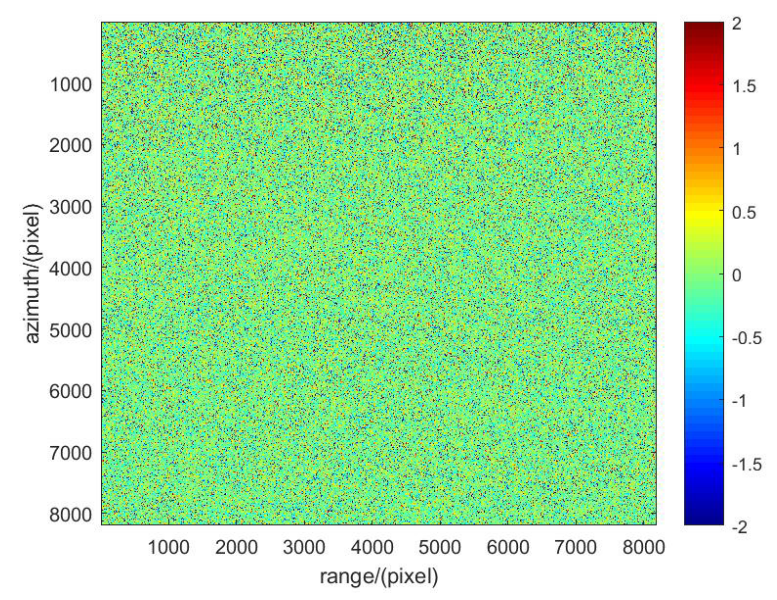

Figure 19. The extracted interferometric phase from the Gaofen-3 along-track InSAR data (size: 8192(azimuth)*8192(range)). 


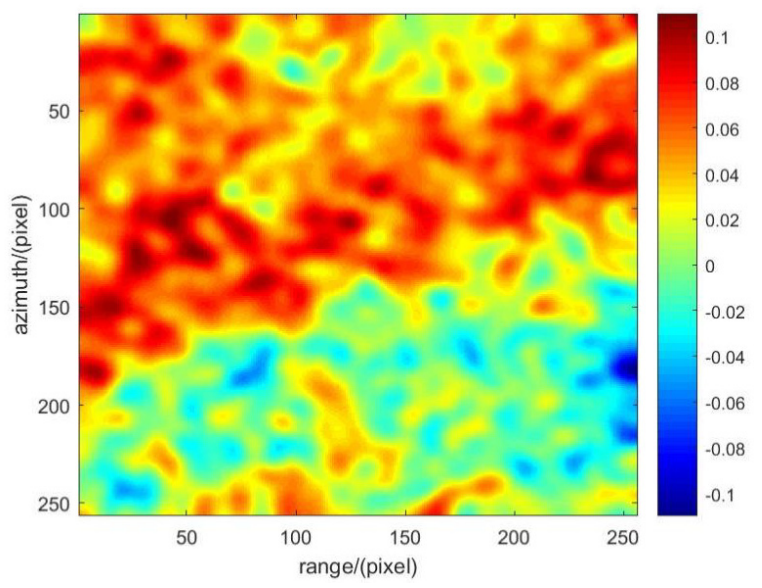

Figure 20. The extracted interferometric phase from the Gaofen-3 along-track InSAR data after 1024 multi-looks and three times $10 * 10$ element boxcar averaging (size: 256(azimuth)*256(range)).

In our experiment, the current field is first calculated with the aforementioned three methods and the results are shown in Figures 21a, 22a and 23a. Then, the calculated current field is input into the M4S software to simulate the corresponding interferometric phases, which are presented in Figures $21 \mathrm{~b}, 22 \mathrm{~b}$ and $23 \mathrm{~b}$. Since the real ocean current flow field data of the observed area are unknown, the simulated interferometric phases with the aforementioned three methods are compared with the extracted interferometric phase, and the corresponding statistics results are shown in Table 12, which can exhibit the performance of these three methods indirectly.

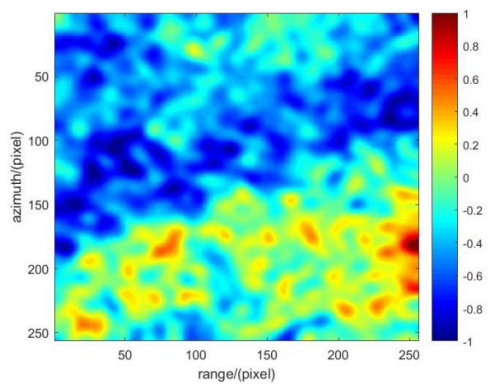

(a)

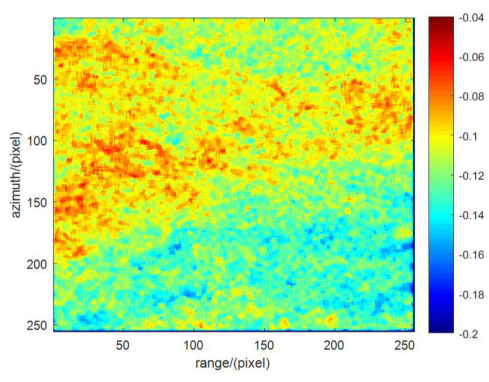

(b)

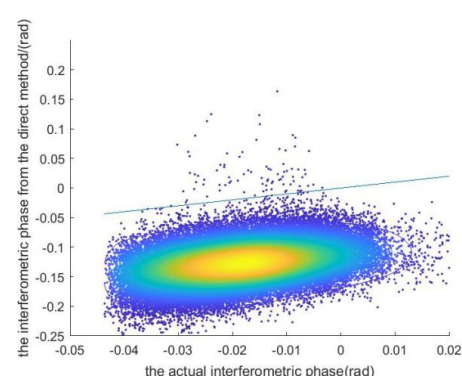

(c)

Figure 21. Results of the direct method: (a) the calculated current filed, (b) the simulated interferometric phase, and (c) the scatter density map of simulated and extracted interferometric phases.

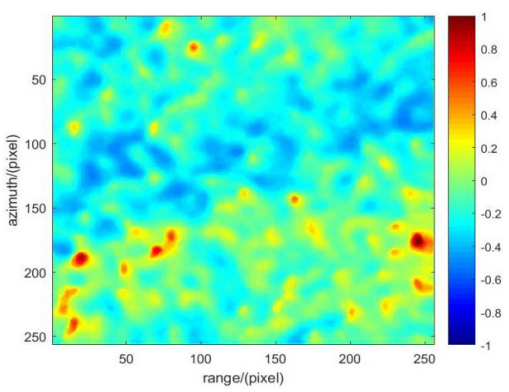

(a)

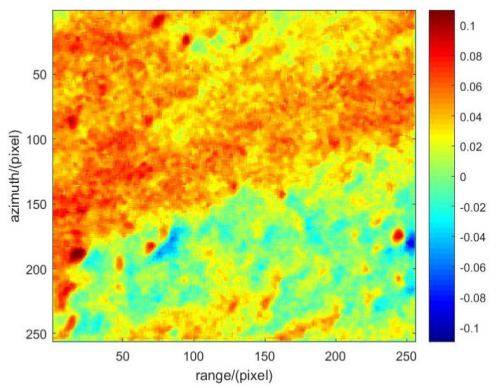

(b)

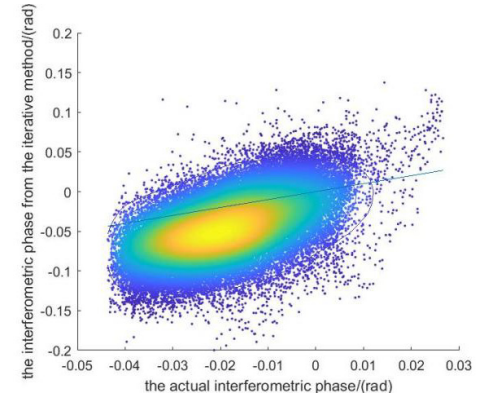

(c)

Figure 22. Results of the iterative method: (a) the calculated current filed, (b) the simulated interferometric phase, and (c) the scatter density map of simulated and extracted interferometric phases. 


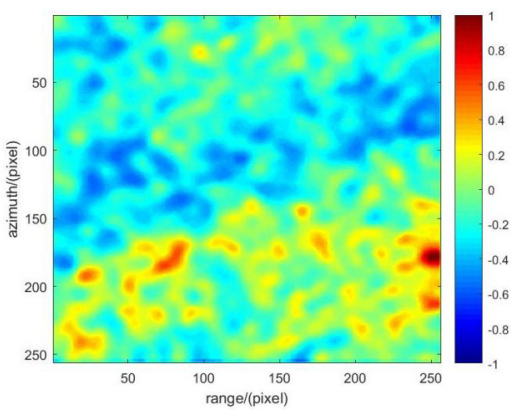

(a)

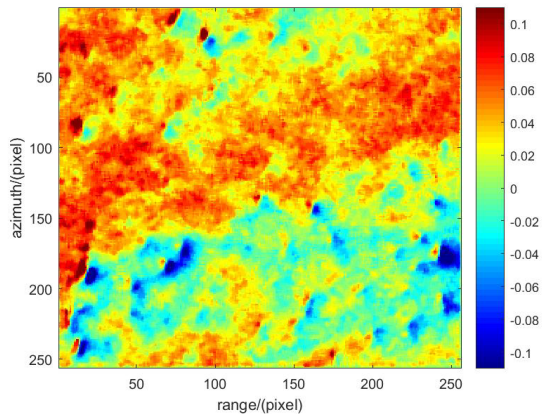

(b)

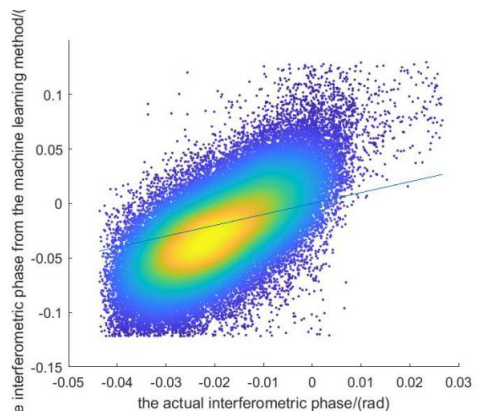

(c)

Figure 23. Results of the proposed method: (a) the calculated current filed, (b) the simulated interferometric phase, and (c) the scatter density map of simulated and extracted interferometric phases.

Table 12. Statistics of the estimation results under different methods.

\begin{tabular}{cccc}
\hline Method & RMSE (rad) & r & Bias (rad) \\
\hline Direct Method & 0.113 & 0.759 & 0.109 \\
Iterative Method & 0.066 & 0.658 & 0.026 \\
Proposed Method & 0.042 & 0.791 & 0.007 \\
\hline
\end{tabular}

Through the aforementioned real-data processing results, it can be found that the proposed method has a significant advantage over the direct method. While comparing to the iterative method, the current velocity estimation performance of the proposed method has a slight improvement. However, the iterative method takes a long time to finish the iteration in real-world applications. This low efficiency can be well solved by the proposed method.

\section{Discussion}

Table 3 summarizes the quantitative indicators of the nine groups of test samples. According to the statistics shown in the table, it can be found that the mean values of the RMSE, r, and bias are $0.022 \mathrm{~m} / \mathrm{s}, 0.768$, and $0.017 \mathrm{~m} / \mathrm{s}$, respectively. Compared with the traditional calculation methods of ocean surface velocity based on the iterative method in Section 3.5, the velocity measurement accuracy of the proposed method increased from $0.084 \mathrm{~m} / \mathrm{s}$ to $0.017 \mathrm{~m} / \mathrm{s}$. This is the most important advantage of the new method proposed in this paper.

In the experiments of the influence of the changing network structure on training results, Table 4 summarizes the quantitative indicators under different epochs. Based on the values in the table, with the rising epoch number, the RMSE decreases before it increases. When the number of the epoch is 300 , the velocity measurement accuracy is the highest and the correlation is the strongest, proving that in the process of network training, the curve will change from under-fitting to over-fitting with the increase in the epoch number. Therefore, there is a need to find the optimal critical value. In addition, Table 5 summarizes the quantitative indicators under the different batch sizes. The values in the table indicate that, when the batch size is 1, the velocity measurement accuracy is the highest and the correlation is the strongest. Moreover, the larger the batch size is, the lower the accuracy is. This is because the number of samples in the training set in this experiment is a small dataset. After reducing the batch size, the convergence was slowed down and the generalization ability of the model was enhanced. Table 6 summarizes the quantitative indicators under different datasets. The larger the number of samples in the dataset is, the higher the accuracy of the current measurement is. This is because the generator can capture more data features with a larger training sample. If the network level remains unchanged, the feature training will be sufficient, so as to produce better prediction results. Table 7 summarizes the quantitative indicators under different learning rates. As shown in 
the table, with the increasing learning rate, the RMSE decreases before it increases. This is because the smaller the learning rate is, the slower the speed of the loss gradient descent is, and the longer the convergence time is. However, for the excessive learning rate, the steps of gradient descent might be too large to capture the optimal value.

In the experiments on how changing system parameters affect the training results, the optimal network based on the above network parameters was selected. Table 9 summarizes the quantitative indicators under different wind velocities. According to the chart, the accuracy of the current measurement is worsened with the increasing wind speed. Based on the M4S software, both wind speed and current rate affect the generation of the interferometric phase. Therefore, the more the wind speed of the test dataset deviates from that of the training set, the greater the error between the velocity obtained through deep learning and the input velocity will be. Table 10 summarizes the quantitative indicators under different numbers of multi-look. The chart suggests that there is little relation between the multiple vision and the accuracy of the current measurement, so that the interference source of the multi-vision can be eliminated from the error analysis in the subsequent experimental studies. Table 11 summarizes the quantitative indicators under different incident angles. The chart reveals that, with the increase in the incident angle, the accuracy of the current measurement climbs up and then declines. This is because the vertical velocity accounts for a large proportion when the incident angle is too small, while the proportion of the horizontal velocity will increase with the incident angle. However, in the case of a large incident angle, the backscattering from the sea surface and the signal-to-noise ratio will decrease. What is more, the accuracy of the current measurement will also decrease.

As for real-data verification, as far as we know, the Gaofen-3 SAR satellite is designed with different operating modes but does not include an ocean current velocity measurement mode. The real data used in our experiment are from the two-channel SARGMTI/SAR-MMTI mode with the radar beam pointing to the sea surface area. Since the ocean current velocity measurement mode is not designed for the Gaofen-3 SAR system, the corresponding cooperation experiments are not carried out and the real ocean current flow field data of the observed area are not known. However, the good news is that the ocean current velocity measurement mode is being designed and demonstrated for a new spaceborne along-track InSAR system in China, which will be launched in the near future. Besides, the corresponding cooperation experiment will also be carried out. By that time, the whole signal processing chain of along-track ATI for ocean current measurements can be demonstrated by real data.

\section{Conclusions}

In this study, we proposed an estimation method for the ocean surface current field velocity based on the CGANs in deep learning. CGANs have already been widely used in the field of image translation, that is 'translating' an input image into a corresponding output image. In our application, the image of interferometric phase can be regarded as the input image, while the image of measured ocean current velocity can be considered as the output image, which fits well the model of CGANs. This is the motivation that we introduce CGANs in the field of ocean current velocity measurement based on along-track InSAR data. The major findings are as follows. First of all, since the measured data from the ocean surface current field were not available, we used the simulated data instead of the measured data. The input current velocity diagrams that we constructed and the corresponding interferometric phase diagrams based on M4S software are used to test the practicability of the method. The network structural parameters have a relatively large impact on the training results. Specifically speaking, when the number of the epoch is 300, the batch size is 1 , dataset samples are 400 , and the learning rate is 0.0002 , the estimation result has the minimum error and the maximum correlation. Based on the optimal network structure, compared with the two traditional methods, that is, the direct method and the iterative method, the proposed method increases the precision of the velocity estimates. Changes in the wind field velocity and incident angle can greatly impact the error of the 
estimation of current velocity, while changes in the multi-look number only have a limited impact. Based on these, the generalization ability of the neural network can be verified.

This study made a novel attempt to apply deep learning to the estimation of current velocity. The experimental results verified the feasibility and effectiveness of the new method. However, there are still some limitations in the proposed method. For instance, no detailed optimization has been made to the network structure, and the number of samples in the dataset is not large enough. Similarly, the types are neither broad enough. These factors are key constraints to higher accuracy. Thus, the future work will focus on the data augmentation, the architecture design, and the algorithm optimization to improve the performance of the system. It is believed that in the near future, deep learning will solve the more complex problems of ocean remote sensing.

Author Contributions: Conceptualization, H.Y., Q.H. and G.J.; methodology, H.Y., Q.H., G.J. and X.X.; software, H.Y. and G.Z.; validation, G.J. and D.Z.; formal analysis, H.Y., Q.H. and D.Z.; investigation, H.Y. and Q.H.; resources, H.Y. and X.X.; data curation, H.Y. and G.J.; writing-original draft preparation, H.Y. and Q.H.; writing—review and editing, G.J. and X.X.; visualization, G.Z.; supervision, D.Z.; project administration, H.Y. and D.Z.; funding acquisition, H.Y. and G.Z. All authors have read and agreed to the published version of the manuscript.

Funding: This research was funded by the National Aerospace Science Foundation of China, grant number 201920052002 and Foundation Strengthening Programme Technical Field Fund, grant number 2020-JCJQ-JJ-386.

Acknowledgments: The authors would like to thank Robert Wang and Mingjie Zheng of Aerospace Information Research Institute, Chinese Academy of Sciences for their support in the along-track InSAR processing.

Conflicts of Interest: The authors declare no conflict of interest.

\section{References}

1. Lian, Z.; Wei, Z.; Wang, Y.; Fang, G. Sea surface height of the China adjacent seas from a variable-grid global ocean circulation model. In Proceedings of the 2011 International Conference on Remote Sensing, Environment and Transportation Engineering, Nanjing, China, 24-26 June 2011; pp. 2463-2466.

2. Elyouncha, A.; Eriksson, L.E.B.; Romeiser, R.; Ulander, L.M.H. Measurements of Sea Surface Currents in the Baltic Sea Region Using Spaceborne Along-Track InSAR. IEEE Trans. Geosci. Remote Sens. 2019, 57, 8584-8599. [CrossRef]

3. Yi, N.; He, Y.; Liu, B. Improved Method to Suppress Azimuth Ambiguity for Current Velocity Measurement in Coastal Waters Based on ATI-SAR Systems. Remote Sens. 2020, 12, 328. [CrossRef]

4. Suchandt, S.; Lehmann, A.; Runge, H. Analysis of Ocean Surface Currents with TanDEM-X ATI: A Case Study in the Baltic Sea. In Proceedings of the 2014 IEEE Geoscience and Remote Sensing Symposium, Quebec City, QC, Canada, 13-18 July 2014; pp. 3918-3921.

5. Wollstadt, S.; López-Dekker, P.; De Zan, F.; Younis, M. Design Principles and Considerations for Spaceborne ATI SAR-Based Observations of Ocean Surface Velocity Vectors. IEEE Trans. Geosci. Remote Sens. 2017, 55, 4500-4519. [CrossRef]

6. Graber, H.C.; Thompson, D.R.; Carande, R.E. Ocean surface features and currents measured with synthetic aperture radar interferometry and HF radar. J. Geophys. Res. C Ocean. 1996, 101, 25813-25832. [CrossRef]

7. Kim, D.; Moon, W.M.; Moller, D.; Imel, D.A. Measurements of ocean surface waves and currents using L- and C-band along-track interferometric SAR. IEEE Trans. Geosci. Remote Sens. 2003, 41, 2821-2832.

8. Siegmund, R.; Bao, M.; Lehner, S.; Mayerle, R. First demonstration of surface currents imaged by hybrid along- and cross-track interferometric SAR. IEEE Trans. Geosci. Remote Sens. 2004, 42, 511-519. [CrossRef]

9. Romeiser, R.; Breit, H.; Eineder, M.; Runge, H.; Flament, P.; De Jong, K.; Vogelzang, J. Current measurements by SAR along-track interferometry from a Space Shuttle. IEEE Trans. Geosci. Remote Sens. 2005, 43, 2315-2323. [CrossRef]

10. Ouchi, K.; Yoshida, T.; Yang, C.S. A Theory of Multiaperture Along-Track Interferometric Synthetic Aperture Radar. IEEE Geosci. Remote Sens. Lett. 2019, 16, 1565-1569. [CrossRef]

11. Ouchi, K.; Yoshida, T.; Yang, C.S. Multi-Aperture Along-Track Interferometric SAR for Estimating Velocity Vector of Ocean Currents. In Proceedings of the IGARSS 2018 IEEE International Geoscience and Remote Sensing Symposium, Valencia, Spain, 22-27 July 2018; pp. 1001-1004.

12. Frasier, S.J.; Camps, A.J. Dual-beam interferometry for ocean surface current vector mapping. IEEE Trans. Geosci. Remote Sens. 2001, 39, 401-414. [CrossRef]

13. Toporkov, J.V.; Perkovic, D.; Farquharson, G.; Sletten, M.A.; Frasier, S.J. Sea surface velocity vector retrieval using dual-beam interferometry: First demonstration. IEEE Trans. Geosci. Remote Sens. 2005, 43, 2494-2502. [CrossRef] 
14. Yu, X.Z.; Chong, J.S.; Hong, W. An iterative method for ocean surface current retrieval by along-track interferometric SAR. Dianzi Yu Xinxi Xuebao/J. Electron. Inf. Technol. 2012, 34, 2660-2665. [CrossRef]

15. Romeiser, R.; Thompson, D.R. Numerical study on the along-track interferometric radar imaging mechanism of oceanic surface currents. IEEE Trans. Geosci. Remote Sens. 2000, 38, 446-458. [CrossRef]

16. Romeiser, R.; Runge, H. Current Measurements in European Coastal Waters and Rivers by Along-Track InSAR. In Remote Sensing of the European Seas; Barale, V., Gade, M., Eds.; Springer: Dordrecht, The Netherland, 2008; pp. 411-422. [CrossRef]

17. Liu, Y.; Li, X.; Ren, Y. A Deep Learning Model for Oceanic Mesoscale Eddy Detection Based on Multi-Source Remote Sensing Imagery. In Proceedings of the IGARSS 2020-2020 IEEE International Geoscience and Remote Sensing Symposium, Waikoloa, HI, USA, 26 September-2 October 2020; pp. 6762-6765.

18. Henley, H.; Berard, A.; Lapisky, E.; Zimmerman, M. Deep Learning in Shallow Water: CNN-based 3D-FLS Target Recognition. In Proceedings of the OCEANS 2018 MTS/IEEE Charleston, Charleston, SC, USA, 22-25 October 2018; pp. 1-7.

19. Goodfellow, I.J.; Pouget-Abadie, J.; Mirza, M.; Xu, B.; Warde-Farley, D.; Ozair, S.; Courville, A.; Bengio, Y. Generative Adversarial Networks. 2014. Available online: http:/ / arxiv.org/abs/1406.2661 (accessed on 10 June 2014).

20. Kwon, H.; Kim, Y.; Yoon, H.; Choi, D. Captcha image generation systems using generative adversarial networks. IEICE Trans. Inf. Syst. 2018, 101, 543-546. [CrossRef]

21. Mirza, M.; Osindero, S. Conditional Generative Adversarial Nets. arXiv 2014, arXiv:1411.1784.

22. Radford, A.; Metz, L.; Chintala, S. Unsupervised representation learning with deep convolutional generative adversarial networks. In Proceedings of the 4th International Conference on Learning Representations, ICLR 2016, San Juan, Puerto Rico, 2-4 May 2016; pp. 1-16.

23. Brock, A.; Donahue, J.; Simonyan, K. Large scale GaN training for high fidelity natural image synthesis. In Proceedings of the 7th International Conference on Learning Representations, ICLR 2019, New Orleans, LA, USA, 6-9 May 2019; pp. 1-35.

24. Zhu, J.Y.; Park, T.; Isola, P.; Efros, A.A. Unpaired Image-to-Image Translation Using Cycle-Consistent Adversarial Networks. In Proceedings of the 2017 IEEE International Conference on Computer Vision (ICCV), Venice, Italy, 22-29 October 2017; pp. 2242-2251.

25. Isola, P.; Zhu, J.Y.; Zhou, T.; Efros, A.A. Image-to-image translation with conditional adversarial networks. In Proceedings of the 2017 IEEE Conference on Computer Vision and Pattern Recognition (CVPR), Honolulu, HI, USA, 21-26 July 2017; pp. 5967-5976.

26. Weng, W.; Zhu, X. INet: Convolutional Networks for Biomedical Image Segmentation. IEEE Access 2021, 9, $16591-16603$. [CrossRef]

27. Mizuochi, H.; Iijima, Y.; Nagano, H.; Kotani, A.; Hiyama, T. Dynamic mapping of subarctic surface water by fusion of microwave and optical satellite data using conditional adversarial networks. Remote Sens. 2021, 13, 175. [CrossRef]

28. Romeiser, R.; Runge, H.; Suchandt, S.; Kahle, R.; Rossi, C.; Bell, P.S. Quality Assessment of Surface Current Fields from TerraSAR-X and TanDEM-X Along-Track Interferometry and Doppler Centroid Analysis. IEEE Trans. Geosci. Remote Sens. 2014, 52, $2759-2772$. [CrossRef]

29. Bishop, C.M. Pattern Recognition and Machine Learning; Springer: Berlin/Heidelberg, Germany, 2006.

30. OSCAR Datasets. Available online: https://podaac-tools.jpl.nasa.gov/drive/files/allData/oscar/preview/L4/oscar_third_deg (accessed on 7 March 2020).

31. Ocean Surface Current Analysis (OSCAR) Third Degree. Available online: https://www.docin.com/p-1939455921.html (accessed on 7 May 2009).

32. Pathak, D.; Krahenbuhl, P.; Donahue, J.; Darrell, T.; Efros, A.A. Context Encoders: Feature Learning by Inpainting. In Proceedings of the 2016 IEEE Conference on Computer Vision and Pattern Recognition (CVPR), Las Vegas, NV, USA, 27-30 June 2016; pp. 2536-2544.

33. Zhang, Q.; Liu, X.; Liu, M.; Zou, X.; Zhu, L.; Ruan, X. Comparative analysis of edge information and polarization on sar-to-optical translation based on conditional generative adversarial networks. Remote Sens. 2021, 13, 128. [CrossRef] 